\title{
Stage-specific metabolic features of differentiating neurons: Implications for toxicant sensitivity
}

\author{
Johannes Delp ${ }^{\mathrm{a}, 1}$, Simon Gutbier ${ }^{\mathrm{a}, 1}$, Martin Cerff ${ }^{\mathrm{b}, 1}$, Christin Zasada ${ }^{\mathrm{c}, 1}$, Sebastian Niedenführ ${ }^{\mathrm{b}}$, \\ Liang Zhao ${ }^{\mathrm{d}}$, Lena Smirnova ${ }^{\mathrm{d}}$, Thomas Hartung ${ }^{\mathrm{d}}$, Hanna Borlinghaus ${ }^{\mathrm{f}}$, Falk Schreiber, ${ }^{\mathrm{f}, \mathrm{g}}$, \\ Jörg Bergemann ${ }^{\mathrm{h}}$, Jochem Gätgens ${ }^{\mathrm{b}}$, Martin Beyss ${ }^{\mathrm{b}}$, Salah Azzouzi ${ }^{\mathrm{b}}$, Tanja Waldmann ${ }^{\mathrm{a}}$, \\ Stefan Kempa ${ }^{\mathrm{c}, 2}$, Katharina Nöh ${ }^{\mathrm{b}, 2}$, Marcel Leist ${ }^{\mathrm{a}, \mathrm{e}, *, 2}$ \\ a In Vitro Toxicology and Biomedicine, Dept Inaugurated by the Doerenkamp-Zbinden Foundation, University of Konstanz, 78457 Konstanz, Germany \\ ${ }^{\mathrm{b}}$ Institute of Bio- and Geosciences, IBG-1: Biotechnology, Forschungszentrum Jülich GmbH, Jülich 52425, Germany \\ ${ }^{\mathrm{c}}$ Max-Delbrück-Center of Molecular Medicine in the Helmholtz Association, Berlin 13125, Germany \\ d Johns Hopkins University, Bloomberg School of Public Health, Center for Alternatives to Animal Testing (CAAT), Baltimore, MD, USA \\ e CAAT-Europe, University of Konstanz, Konstanz 78457, Germany \\ ${ }_{\mathrm{f}}^{\mathrm{f}}$ Department of Computer and Information Science, University of Konstanz, Konstanz, Germany \\ ${ }^{\mathrm{g}}$ Faculty of Information Technology, Monash University, Melbourne, Australia \\ ${ }^{\mathrm{h}}$ Department of Life Sciences, Albstadt-Sigmaringen University of Applied Sciences, Sigmaringen, Germany
}

Keywords:

Developmental neurotoxicity

Metabolomics

Energy metabolism

Metabolic flux

${ }^{13} \mathrm{C}$ labeling experiment

Oxygen consumption

\begin{abstract}
A B S T R A C T
Developmental neurotoxicity (DNT) may be induced when chemicals disturb a key neurodevelopmental process, and many tests focus on this type of toxicity. Alternatively, DNT may occur when chemicals are cytotoxic only during a specific neurodevelopmental stage. The toxicant sensitivity is affected by the expression of toxicant targets and by resilience factors. Although cellular metabolism plays an important role, little is known how it changes during human neurogenesis, and how potential alterations affect toxicant sensitivity of mature vs. immature neurons. We used immature (d0) and mature (d6) LUHMES cells (dopaminergic human neurons) to provide initial answers to these questions. Transcriptome profiling and characterization of energy metabolism suggested a switch from predominantly glycolytic energy generation to a more pronounced contribution of the tricarboxylic acid cycle (TCA) during neuronal maturation. Therefore, we used pulsed stable isotope-resolved metabolomics (pSIRM) to determine intracellular metabolite pool sizes (concentrations), and isotopically nonstationary ${ }^{13} \mathrm{C}$-metabolic flux analysis (INST ${ }^{13} \mathrm{C}$-MFA) to calculate metabolic fluxes. We found that d0 cells mainly use glutamine to fuel the TCA. Furthermore, they rely on extracellular pyruvate to allow continuous growth. This metabolic situation does not allow for mitochondrial or glycolytic spare capacity, i.e. the ability to adapt energy generation to altered needs. Accordingly, neuronal precursor cells displayed a higher sensitivity to several mitochondrial toxicants than mature neurons differentiated from them. In summary, this study shows that precursor cells lose their glutamine dependency during differentiation while they gain flexibility of energy generation and thereby increase their resistance to low concentrations of mitochondrial toxicants.
\end{abstract}

\section{Introduction}

Developmental neurotoxicity (DNT) testing is currently undergoing a major transition from expensive, time consuming animal based studies to cell-based assays (Schmidt et al., 2017). Most in vitro tests model a narrow window of development, and they reflect only one or

\footnotetext{
Abbreviations: aKG, $\alpha$-ketoglutarate; AA, amino acid; BM, biomass; CCM, central carbon metabolism; CDW, cell dry weight; DNT, Developmental neurotoxicity; ECAR, extracellular acidification rate; ESI, Electrospray ionization; FCCP, Carbonyl cyanide-4-(trifluoromethoxy)phenylhydrazone; GC-MS, gas chromatography-mass spectrometry; GO, gene ontology; INST ${ }^{13} \mathrm{C}-\mathrm{MFA}$, isotopically non-stationary ${ }^{13} \mathrm{C}$ metabolic flux analysis; IVT, in vitro transcription; LUHMES, Lund human mesencephalic cells; ME, malic enzyme; MID, mass isotopomer distribution; OCR, oxygen consumption rate; PC, pyruvate carboxylase; PCA, principal component analysis; PDH, pyruvate dehydrogenase; pSIRM, pulsed stable isotope-resolved metabolomics; RQI, RNA quality indicator; SD, standard deviation; SEM, standard error of the mean; TCA, tricarboxylic acid cycle

* Corresponding author at: In Vitro Toxicology and Biomedicine, Dept Inaugurated by the Doerenkamp-Zbinden Foundation, University of Konstanz, Konstanz 78457, Germany.

E-mail address: marcel.leist@uni-konstanz.de (M. Leist).

1 Shared first author.

${ }^{2}$ Shared last author.
} 
few neurodevelopmental processes. Therefore, in order to obtain toxicity predictions with a high confidence level, several assays will have to be combined to a test battery (Aschner et al., 2017; Fritsche et al., 2017). To cover all actions of DNT toxicants, it is important to focus not only on specific disturbances of key developmental processes. Also blunt cytotoxicity can play a role, if it is specific for a subpopulation of cells that is only present during a particular phase of organ development. For instance exposure to the general cytotoxicant 5-flurouracil can affect facial and hindlimb development in specific ways (Lau et al., 2001) and exposure to the cell cycle toxicant methylazoxymethylacetat (MAM) is a standard tool to trigger structural and functional DNT (Penschuck et al., 2006; Kadereit et al., 2012; van Thriel et al., 2012). The understanding of specific sensitivities to cytotoxicants (Smirnova et al., 2015) and of the underlying biological and metabolic basis will be important for mechanistic validation of test systems and for the assessment of their biological relevance (Leist et al., 2008a; Leist et al., 2008b; Leist et al., 2012; Hartung et al., 2013).

In the early fetus, most cells are highly proliferative in order to generate body growth. For instance, neural precursor cells (NPC) generate abundant progeny to create the nervous system. Some DNT test methods are based on the differentiation of pluripotent cells to NPCs (Balmer et al., 2012; Waldmann et al., 2017). Others use specific precursors of the peripheral nervous system to study their function (Zimmer et al., 2012; Dreser et al., 2015), or such cells are being used to examine for instance astrocytogenesis (Kleiderman et al., 2016a; Kleiderman et al., 2016b). Monitoring of many of the diverse proliferation and differentiation steps in the nervous system is a pivotal aspect of DNT testing (van Thriel et al., 2012; Smirnova et al., 2014). Until now, such monitoring is mainly descriptive, based on the quantification of markers for various cell populations. A step towards a more mechanistic understanding of transitions between cells are time-resolved studies of transcriptional markers (Balmer et al., 2014; Rempel et al., 2015; Shinde et al., 2016; Shinde et al., 2017). A further important step requires detailed studies on metabolic changes (Teslaa and Teitell, 2015; Cabre et al., 2016; Jang et al., 2016; Esteban-Martinez et al., 2017; Sa et al., 2017).

Differentiation of neurons implies cell cycle arrest and cessation of proliferation. Besides the obvious morphological changes (e.g. neuritogenesis), this process is accompanied by a strong alteration in the metabolic phenotype (Shyh-Chang et al., 2013; Agostini et al., 2016). Proliferating cells have a higher demand of glucose and an increased glycolytic rate, which contributes to the generation of ATP (Vander Heiden et al., 2009; Fan et al., 2013). In addition, glutamine is taken up to generate essential metabolites for growth (Birsoy et al., 2015; Sullivan et al., 2015) and to sustain oxidative phosphorylation for ATP production (Fan et al., 2013). Post-mitotic neurons in brain tissue cope with a different metabolic situation: they have a higher basal energy demand, but they don't have to generate new cells. Under these conditions, adult neurons metabolize glucose to $\mathrm{CO}_{2}$ through oxidative phosphorylation via the tricarboxylic acid cycle (TCA) (Magistretti and Allaman, 2015). The use of glutamate and glutamine as alternative carbon source is limited to few special situations (McKenna, 2007).

Most available data on the central carbon metabolism (CCM) of cells are derived either from cancer cells or from tissue measurements, and there are only few studies comparing different stages of the same cell line. Some data on changes of the metabolic phenotype during cellular differentiation and de-differentiation have been reported for induced pluripotent stem cells and for neural progenitor lines (Folmes et al., 2011; Folmes et al., 2012; Zhang et al., 2012; Sa et al., 2017).

Several other studies characterized metabolic changes when cells switched from exponential growth to growth arrest. For example, two studies reported differences between $\mathrm{CHO}$ cells in either growth or stationary phases. Changes occurring during the transition from proliferation to a stationary state were mainly observed in the pentose phosphate pathway, glycolysis and the carbon supply to the TCA (anaplerosis) (Ahn and Antoniewicz, 2011; Ahn and Antoniewicz,
2013). A further study compared the metabolic differences upon differentiation of neuronal precursor cells to astrocytes (Sa et al., 2017). All these data sets suggest distinct metabolic differences between proliferating and differentiated cells. The metabolism of cancer cells and of other proliferating cells shows many similarities, presumably due to their shared need to generate new biomass during proliferation (Vazquez et al., 2010; Zhang et al., 2012; Schuster et al., 2015). Recent data from cultured murine cortical neurons suggest that changes in glycolysis and in the glutamine-glutamate pathway also may play a key role in the process of neuronal differentiation (Agostini et al., 2016). However, there is still a need for detailed information about metabolic alterations during human neuronal differentiation, not at least because this feature might affect the toxicant sensitivity of these cells in different stages of neuronal maturation.

There are multiple examples for cell-type specific toxicity. For a variety of toxicants it has been observed that proliferating progenitor cells are more susceptible to undergo cell death following toxicant exposure than their differentiated progeny (Ninomiya et al., 1997; Gartlon et al., 2006; Hu and Xuan, 2008; Tong et al., 2017). It has been discussed intensively, whether this might be due to their different levels of antiapoptotic proteins, their differences in drug transporter expression, or the replication machinery. However, there are also many situations, when differentiated cells are more toxicant-sensitive than their precursors. Examples are the paracetamol toxicity to primary hepatocytes vs. immature hepatic precursors (Sengupta et al., 2014) or the neurotoxicity of the parkinsonian toxicant $\mathrm{MPP}^{+}$(Schildknecht et al., 2009; Schildknecht et al., 2015; Smirnova et al., 2016). Further examples are astrocyte-specific toxicants such as fluoroacetate, fluorocitrate or 6-aminonicotinamide that selectively affect the energy metabolism of astrocytes (Swanson and Graham, 1994; Haghighat and McCandless, 1997).

In summary, such altered susceptibilities may depend on a differential expression of targets, receptors, transporters or metabolizing enzymes. Alternatively, altered metabolic states may be responsible for cell stage specific toxicity. To obtain more information on this issue, the present study sets out to characterize metabolic changes upon neuronal differentiation. We used conditionally immortalized dopaminergic neuronal precursor cells (LUHMES cells), which proliferate in their basal condition (d0), and which can be differentiated to post-mitotic dopaminergic cells within 6 days (d6). Proliferating cells express the neuronal precursor markers SOX2, PAX3, BRN3A, ASCL1 and nestin. Upon differentiation, gene expression changes and genes coding for synaptic proteins (e.g. GRIN1, DLG4) and dopaminergic markers like tyrosine hydroxylase (TH), dopamine D2-receptor (DRD2) and the dopamine transporter (DAT) are upregulated. The differentiation process is characterized by its uniformity and synchronization (Lotharius et al., 2005; Scholz et al., 2011; Krug et al., 2013a; Krug et al., 2014). This model system is therefore suitable for biochemical studies that require large numbers of cells at the same differentiation stage. We made use of this model to measure the role of media constituents and the susceptibility to few selected toxicants. To obtain a better understanding of the metabolic states of LUHMES cells, we profiled extracellular key nutrients/products by their rates of consumption/production. Furthermore, we combined the use of stable isotope tracers with state-of-theart metabolomics and fluxomics approaches. Especially, we applied mass spectrometry based pulsed stable isotope-resolved metabolomics (pSIRM) and isotopically non-stationary ${ }^{13} \mathrm{C}$ metabolic flux analysis (INST ${ }^{13} \mathrm{C}$-MFA) for the measurement of intracellular metabolite pool sizes and dynamic labeling incorporation profiles as well as the calculation of intracellular metabolic fluxes.

\section{Material and methods}

\subsection{Cell culture}

Handling of LUHMES human neuronal precursor cells was performed as previously described in detail (Lotharius et al., 2005; Scholz 
et al., 2011; Krug et al., 2014). Briefly, the conditionally-immortalized cells (expressing a v-myc transgene under the control of a tet-off system maintained in proliferation medium (PM: advanced DMEM/F12, $2 \mathrm{mM}$ L-glutamine, $1 \times \mathrm{N} 2$ supplement (Invitrogen), and $40 \mathrm{ng} / \mathrm{ml} \mathrm{FGF-2)} \mathrm{in}$ a $5 \% \mathrm{CO}_{2} / 95 \%$ air atmosphere at $37{ }^{\circ} \mathrm{C}$. Passaging was three times a week. For differentiation, 8 million cells were seeded in a Nunclon T175 tissue culture flask in PM. After $24 \mathrm{~h}$, medium was changed to differentiation medium (DM: advanced DMEM/F12, $2 \mathrm{mM}$ L-glutamine, $1 \times \mathrm{N} 2,2.25 \mu \mathrm{M}$ tetracycline, $1 \mathrm{mM}$ dibutyryl $3^{\prime}, 5^{\prime}$-cyclic adenosine monophosphate (cAMP) and $2 \mathrm{ng} / \mathrm{ml}$ recombinant human glial cell derived neurotrophic factor (GDNF)). At $48 \mathrm{~h}$ later, cells were trypsinised, and seeded in a density of $1.8 * 10^{5} \mathrm{cells} / \mathrm{cm}^{2}$ on dishes precoated with $50 \mu \mathrm{g} / \mathrm{ml}$ poly-L-ornithine (PLO) and $1 \mu \mathrm{g} / \mathrm{ml}$ fibronectin in DM. On day 4 of differentiation, medium was replaced.

\subsection{Standard experimental setup}

To generate d6 cells, LUHMES differentiated for 2 days were replated at a density of $1.5 * 10^{5}$ cells per $\mathrm{cm}^{2}$ in DM and left to differentiate for additional 4 days. To obtain d0 cells, proliferating cells were seeded at a density of $6.5 * 10^{4}$ cells per $\mathrm{cm}^{2}$ in PM on PLO/fibronectin coated dishes. At $24 \mathrm{~h}$ after seeding they were used for experiments.

\subsection{Determination of cell numbers and cellular growth}

Cells were seeded at standard density into 96 well plates in the respective medium, containing the indicated supplements. The cell number was assessed several times after seeding by live cells imaging, using Hoechst-33342 and calcein-AM dyes, which were applied $30 \mathrm{~min}$ prior to imaging. Automated microscopy was used to image, and subsequent algorithmic analysis was used to determine viability: double positive cells were counted as live cells whereas cells only positive for $\mathrm{H}-33342$ were counted as dead. The number of live cells was expressed relatively to the amount of seeded cells.

\subsection{Determination of glucose, lactate and pyruvate in cell culture medium}

Glucose and lactate were determined by a commercially available colorimetric cuvette assay (GLU-142 and LAC-142, respectively. Diaglobal, Berlin, Germany). In brief, glucose or lactate was converted by enzymes into colorimetric products which were quantified by spectrophotometric measurements and comparison to calibration standards. Pyruvate was quantified through enzymatic conversion to lactate for which NADH was used up. The decrease in NADH was quantified spectrophotometrically $(\lambda=340 \mathrm{~nm})$ and compared to calibration standards.

\subsection{Determination of total cellular protein}

Total protein content and weight of d0 and $\mathrm{d} 6$ cells was measured in cell populations (about $5 \times 10^{7}$ cells) detached by trypsinization. The cells were washed, counted and weighed (wet weight). After that, cells were lysed in $1 \mathrm{M} \mathrm{NaOH}$ for $24 \mathrm{~h}$, then protein was quantified (BCA assay kit, according to the manufacturer's instructions (Pierce/Thermo Fisher Scientific, Rockford, IL, USA)).

\subsection{Cell viability measurement}

\subsubsection{Resazurin measurement}

Metabolic activity was detected by a resazurin assay. Briefly, resazurin solution was added to the cell culture medium to obtain a final concentration of $2 \mu \mathrm{g} / \mathrm{ml}$. After incubation for $60 \mathrm{~min}$ at $37^{\circ} \mathrm{C}$, the fluorescence signal was measured at an excitation wavelength of $530 \mathrm{~nm}$, using a $590 \mathrm{~nm}$ long-pass filter to record the emission. After background subtraction, fluorescence values were normalized by setting fluorescence values of untreated control wells to $100 \%$.

\subsection{2. $L D H$ release}

LDH activity was detected separately in the supernatant and cell homogenate. Cells were lysed in PBS/0.1\% Triton X-100 (V/V) for $1 \mathrm{~h}$. $10 \mu \mathrm{l}$ of sample was added to $100 \mu \mathrm{l}$ of reaction buffer containing NADH $(100 \mu \mathrm{M})$ and sodium pyruvate $(600 \mu \mathrm{M})$ in KPP-buffer. Absorption at $340 \mathrm{~nm}$ was measured at $37^{\circ} \mathrm{C}$ in $1 \mathrm{~min}$ intervals over a period of $15 \mathrm{~min}$. The slope of the absorption intensity was calculated. The ratio of $\mathrm{LDH}_{\text {supernatant }} / \mathrm{LDH}_{\text {total }}$ was calculated using the slopes of supernatant and homogenate. LDH release was expressed in percent (Krug et al., 2014).

\subsubsection{ATP determination}

To determine intracellular ATP, cells grown in 24-well plates were scratched and sonicated in PBS-buffer and boiled at $95^{\circ} \mathrm{C}$ for $10 \mathrm{~min}$ followed by centrifugation at $10,000 \mathrm{~g}$ for $5 \mathrm{~min}$ for the removal of cell debris. For the detection of ATP levels, a commercially available ATP assay reaction mixture (Sigma, Steinheim, Germany), containing luciferin and luciferase, was used. Fifty microliters sample and $100 \mu \mathrm{l}$ of assay-mix were added to a black 96-well plate. Standards were prepared by serial dilutions of ATP disodium salt hydrate (Sigma, Steinheim, Germany) to obtain final concentrations ranging from $1000 \mathrm{nM}$ to $7.8 \mathrm{nM}$. Determination of protein concentration was performed by using a BCA protein assay kit (Pierce/Thermo Fisher Scientific, Rockford, IL, USA) (Latta et al., 2000; Volbracht et al., 2001; Schildknecht et al., 2009).

\subsection{Extracellular amino acid and ammonia determination}

Medium was collected after the indicated time and mixed with $10 \%$ (W/V) 5-sulfosalicylic acid (SSA) in a volume ratio of 4 to 1 to obtain a final SSA concentration of $2 \%$ in biological triplicates. After shaking for $15 \mathrm{~min}$ at $1400 \mathrm{rpm}$ at $4^{\circ} \mathrm{C}$ in an Eppendorf Thermomix (Hamburg, Germany), the solution was centrifuged for $15 \mathrm{~min}$ at $16,000 \mathrm{~g}$ at $4{ }^{\circ} \mathrm{C}$ to separate the supernatant from the protein precipitations. For amino acid analysis, a 1 to 1 mixture of supernatant and sample dilution buffer (pH 2.2, $0.12 \mathrm{M}$ ) (Sykam, Fürstenfeldbruck, Germany) was prepared. The amino acids were then quantified using a Sykam S433 Amino acid analyzer (Sykam, Fürstenfeldbruck, Germany). Shortly, amino acids and ammonia were separated by HPLC and subsequent post-column derivatization with ninhydrin. Samples were directly injected in a volume of $100 \mu \mathrm{l}$. Chromatography was performed using a lithium based anion exchange column loaded with spherical polystyrene resin $(7 \mu \mathrm{m}$ diameter, 10\% crosslinks, cat\# 5125022). Elution was performed using buffers with increasing $\mathrm{pH}$ and ion strength $(\mathrm{pH} 2.9 \rightarrow \mathrm{pH} 12$; buffer concentration $0.12 \mathrm{M}$ to $0.45 \mathrm{M}$ ), supported by a temperature gradient. Absorbance of the reaction products was quantified at $440 \mathrm{~nm}$ (intermediate product; quantifies cysteine and proline) or $570 \mathrm{~nm}$ (quantifies all other amino acids). Amino acid concentrations were determined relative to a reference standard using the area under the peak method in the ChromStar 7 software (SCPA, Weyhe-Leehste, Germany) (Efremova et al., 2017). Ammonia was quantified following the same protocol.

\subsection{Determination of cell-specific extracellular metabolic rates}

Cell stage-specific extracellular (net) metabolic rates for proliferating $\mathrm{d} 0$ cells, i.e., growth rate $\left[\mathrm{h}^{-1}\right]$, uptake and secretion rates $\left[\mathrm{nmol} \times 10^{6}\right.$ cells $^{-1} \times \mathrm{h}^{-1}$ ], were jointly estimated from extracellular concentration time courses of detectable carbon sources present in the medium and viable cell numbers $(0-12 \mathrm{~h})$, using first order ordinary differential equations while accounting for degradation of glutamine (Gln) with a degradation rate $\mathrm{k}_{\mathrm{Gln}}=2.0 \cdot 10^{-3}\left[\mathrm{~h}^{-1}\right]$ (Ahn and Antoniewicz, 2011). Confidence intervals of the rate estimates were derived by parametric bootstrapping with 5000 samples. For post-mitotic (non-growing) d6 cells, cell stage-specific extracellular rates were determined by linear regression of the extracellular concentration time courses $(0-24 \mathrm{~h})$ and normalization with the average cell concentration. 
Cell concentrations were calculated from cell numbers based on a volume of the liquid phase of $2 \mathrm{ml}$ per well and accounting for volume losses of $1.5-4.5 \%$ due to evaporation. Standard deviations were obtained using Gaussian error propagation. Calculations were performed with Matlab (The MathWorks Inc., Natwick, USA).

\subsection{Seahorse measurements}

Cells were plated $24 \mathrm{~h}$ prior to the experiment in PLO/fibronectincoated "Seahorse 24 well plates" at a density of 100,000 cells (d6 cells) per well or 60,000 cells per wells (d0 cells) in their normal media.

\subsubsection{Mitostress test}

One hour prior to analysis, medium was changed to Seahorse $\mathrm{XF}$ base medium, supplemented with $18 \mathrm{mM}$ glucose, $2 \mathrm{mM}$ glutamine and $1 \mathrm{mM}$ pyruvate. Mitochondrial oxygen consumption was assessed (i) basally and after addition of (ii) $1 \mu \mathrm{M}$ oligomycin, (iii) $1.5 \mu \mathrm{M}$ carbonyl cyanide-4-(trifluoromethoxy)phenylhydrazone (FCCP) and (iv) $0.5 \mu \mathrm{M}$ rotenone with $0.5 \mu \mathrm{M}$ antimycin A. Mitochondrial parameters were calculated as described in results. After assaying mitochondrial function, cells were counted to normalize oxygen consumption relative to total cell number.

\subsubsection{Glycostress test}

One hour prior to analysis, medium was changed to Seahorse XF base medium, supplemented with no glucose, $2 \mathrm{mM}$ glutamine and $1 \mathrm{mM}$ pyruvate. Extracellular acidification rate was assessed (i) basally and after addition of (ii) $10 \mathrm{mM}$ glucose, (iii) $1 \mu \mathrm{M}$ oligomycin and (iv) $50 \mathrm{mM}$ 2-deoxyglucose. Glycolytic parameters were calculated as described in results. After assaying glycolytic function, cells were counted as described above to normalize extracellular acidification relative to total cell number.

\subsection{Non-targeted metabolomics analysis}

D0 and d6 cells were cultured according to standardized culture conditions. Metabolite extraction and measurement was performed as described earlier (Krug et al., 2014). In brief, cells were washed with ice cold PBS and extracted with $80 \% / 20 \% \mathrm{MeOH} /$ water solution. Supernatants were evaporated to dryness at room temperature in a Speedvac concentrator (Savant, Thermo Fisher Scientific, Waltham, MA, USA). The dried samples were reconstituted and measured by liquid chromatography using Agilent 1260 high performance liquid chromatography system with a Cogent Diamond Hydride column (MicroSol, Eatontown, NJ, USA), followed by a 6520 accurate-mass Q-TOF LC-MS system (Agilent) equipped with a dual electrospray (ESI) ion source operated in negative-ion mode for metabolic profiling. Raw data were processed with Mass Hunter Qualitative Analysissoftware (Agilent, version 5.0) (Krug et al., 2014).

\subsection{Pulsed stable isotope-resolved metabolomics (pSIRM)}

LUHMES cells were cultured as described above. To assure standardized conditions, fresh PM or DM (containing $5.5 \mathrm{mM}$ glucose) was added $4 \mathrm{~h}$ prior to start of the labeling $(\mathrm{t}=-4 \mathrm{~h})$. At $\mathrm{t}=0 \mathrm{~h}$, medium was changed again to glucose-free and glutamine-free AdvDMEM/F12 medium (either supplemented with $5.5 \mathrm{mM}\left[\mathrm{U}^{13} \mathrm{C}\right]$ glucose plus $2 \mathrm{mM}$ [U- $\left.{ }^{12} \mathrm{C}\right]$ glutamine or $5.5 \mathrm{mM}\left[\mathrm{U}_{-}{ }^{12} \mathrm{C}\right]$ glucose plus $2 \mathrm{mM}$ [U- $\left.{ }^{13} \mathrm{C}\right]$ glutamine). Both tracers were purchased from Campro Scientific (Berlin, Germany, cat. numbers CS01-183_417 (glc) and CS01-183_434 (gln), both $99 \%$ atom-\%). After $0,2,8,16,30,60,180,360,480,1440$ and $2880 \mathrm{~min}$, samples were harvested according to the pSIRM protocol for adherent cells in 6-well plates (Pietzke et al., 2014). Every biological replicate consisted of two pooled wells of independent differentiations. Cell extracts were stored in 50\%-Methanol (Sigma) until further sample processing. The extraction solvent methanol had been supplemented with cinnamic acid (Sigma, $2 \mu \mathrm{g} / \mu \mathrm{l})$ as an internal extraction standard control.

Extracts were supplemented with chloroform (Sigma) for methanol:chlorofom:water extraction (5:2:1 v/v/v) and incubated for $30 \mathrm{~min}$ at $4{ }^{\circ} \mathrm{C}$ on a rotary wheel, and subsequently centrifuged for phase separation (15 min, maximum speed). Equal volumes of polar phase were collected from each sample separately and dried under vacuum. Extracts were stored at $-25^{\circ} \mathrm{C}$ until continuing with the preparation for GC-MS measurement.

\subsection{GC-MS pSIRM sample preparation}

Derivatization was carried out as described with modifications (Kempa et al., 2009). First, dried extracts were dissolved in $20 \mu \mathrm{l}$ of methoxyamine hydrochloride solution (Sigma, $40 \mathrm{mg} / \mathrm{ml}$ in pyridine (Roth)) and incubated for $90 \mathrm{~min}$ at $30^{\circ} \mathrm{C}$ under constant shaking. In a second phase samples were incubated with $80 \mu \mathrm{l}$ of $N$-methyl- $N$-[trimethylsilyl]trifluoroacetamide (MSTFA; Machery-Nagel, Dueren, Germany) at $37^{\circ} \mathrm{C}$ for $45 \mathrm{~min}$. The in-house alkane standards were added prior to the MSTFA for retention time analysis ( $10 \mu \mathrm{l} / 1 \mathrm{ml}$ MSTFA). The extracts were centrifuged for $10 \mathrm{~min}$ at $10,000 \mathrm{~g}$, aliquoted and transferred in $30 \mu \mathrm{l}$ into glass vials (Th. Geyer, Berlin, Germany) for GC-MS measurement.

Formulation of GC-MS standards for retention index determination, as well as for identification and quantification of metabolites are described in (Pietzke et al., 2014). Identification and quantification of standards was performed parallel with the samples. The quantificationstandard is an in-lab developed mixture, composed of 65 compounds with known quantities (Pietzke et al., 2014). The primary stock is prepared in eight concentrations from 1:1 to 1:200. All eight standards were extracted, derivatized and measured in parallel to the samples. For each batch of measurements a fresh aliquot of quantification standards has been prepared.

\subsection{GC-MS measurements of pSIRM experiments}

Metabolite analysis was performed on a gas chromatography coupled to time of flight mass spectrometer (Pegasus III- TOF-MS-System, LECO Corp., St. Joseph, MI, USA), complemented with an auto-sampler (MultiPurpose Sampler 2 XL, Gerstel, Mülheim an der Ruhr, Germany). The samples and quantification standards were injected in split (split 1:5, injection volume $1 \mu \mathrm{l}$ ) and splitless mode (injection volume $1 \mu \mathrm{l}$ ) in a temperature-controlled injector (CAS4, Gerstel) with a baffled glass liner (Gerstel). The following temperature program was applied during sample injection: initial temperature of $80^{\circ} \mathrm{C}$ for $30 \mathrm{~s}$ followed by a ramp with $12{ }^{\circ} \mathrm{C} / \mathrm{min}$ to $120^{\circ} \mathrm{C}$ and a second ramp with $7{ }^{\circ} \mathrm{C} / \mathrm{min}$ to $300^{\circ} \mathrm{C}$ and final hold for $2 \mathrm{~min}$. Gas chromatographic separation was performed on an Agilent $6890 \mathrm{~N}$ (Agilent, Santa Clara, CA, USA), equipped with a VF-5 ms column of 30-m length, 250- $\mu \mathrm{m}$ inner diameter, and 0.25- $\mu \mathrm{m}$ film thickness (Varian, Palo Alto, CA, USA). Helium was applied as carrier gas with a flow rate of $1.2 \mathrm{ml} / \mathrm{min}$. Gas chromatography was performed with the temperature gradient: 2-min heating at $70{ }^{\circ} \mathrm{C}$, first temperature gradient with $5{ }^{\circ} \mathrm{C} / \mathrm{min}$ up to $120^{\circ} \mathrm{C}$ and hold for $30 \mathrm{~s}$; subsequently, a second temperature step of $7{ }^{\circ} \mathrm{C} / \mathrm{min}$ up to $200{ }^{\circ} \mathrm{C}$ and a final ramp of $12{ }^{\circ} \mathrm{C} / \mathrm{min}$ up to $320^{\circ} \mathrm{C}$ with a hold time of $2 \mathrm{~min}$. The spectra were recorded in a mass range of 60 to $600 \mathrm{U}$ with $20 \mathrm{spectra} / \mathrm{s}$ at a detector voltage of $1750 \mathrm{~V}$.

\subsection{Data analysis of pSIRM experiments}

File processing and analysis was performed with the vendor software ChromaTOF Version 4.42 (LECO). Processing parameters: baseline offset of 1 , peak width of $4 \mathrm{~s}$, signal/noise of 20 , and peak smoothing of 11 data points. Retention index determination and metabolite annotation was performed with the in-house developed software MAUI-VIA (Kuich et al., 2014). Quantification of metabolites was performed by 
external calibration as described in (Kempa et al., 2009). Absolute quantities were normalized to internal extraction standard controls and further quality parameters. Mass isotopomer distributions (MID) for each metabolite and measurement were exported by an extended module of MAUI-VIA and manually validated prior the implementation for metabolic flux analysis. Selected mass ranges for MIDs have been described in (Pietzke et al., 2014).

\subsection{Metabolic flux analysis (MFA): modeling and computational procedures}

A metabolic network model was constructed for LUHMES d0 cells using metabolic reaction databases (www.genome.jp/kegg, www. biocyc.org) and literature knowledge (Duarte et al., 2007; Ahn and Antoniewicz, 2011; Nicolae et al., 2014; Carinhas et al., 2016). The model includes all major metabolic pathways of central carbon metabolism (glycolysis, pentose phosphate pathway, TCA, anaplerosis), and condensed versions of amino acid biosynthesis, lactate- and fatty acid metabolism pathways (acetyl-CoA as precursor). Cellular growth is modelled by a lumped biomass equation. The biomass equation accounts for synthesis of proteins, lipids, DNA, RNA and carbohydrates. Biomass coefficients from (Sheikh et al., 2005) $\left[\mathrm{mmol} / \mathrm{g}_{\mathrm{CDW}}\right]$ were converted to $\left[\mathrm{nmol} / 10^{6}\right.$ cells] with the conversion factor $0.14 \mathrm{mg}_{\mathrm{cdw}} /$ $10^{6}$ cells estimated from total protein content of LUHMES cells $(0.07$ $\mathrm{mg}_{\text {Protein }} / 10^{6}$ cells), and the assumption that the total cell mass consists of $\sim 50 \%$ proteins (Norton and Poduslo, 1971). Although LUHMES cells undergo morphological changes during differentiation, both stages were comparable in weight/cell and protein/cell, hence comparable total cell sizes can be assumed (data not shown). Metabolic reactions were allocated to one of three compartments (cytosol, mitochondria and extracellular space). Transport reactions of intra- and extracellular amino acids were specified according to the medium specification and extracellular rate measurements. Catabolic reactions of amino acids were modelled as lumped reactions fueling succinate, glutamate and acetyl-CoA pools. Carbon atom transitions were formulated for all metabolic reactions. In case of C-symmetric reactants, scrambling reactions were specified assuming equal fluxes for each variant. In total, the model consists of 72 reactions (51 unidirectional, 21 reversible), as well as 32 balanced intracellular, and 11 unbalanced extracellular metabolite pools. The metabolic network model used for ${ }^{13} \mathrm{C}-\mathrm{MFA}$ along with the carbon atom transitions is given in Suppl. Table 3.

With this model, isotopically non-stationary (INST) ${ }^{13} \mathrm{C}-\mathrm{MFA}$ was performed. To this end, the high-performance simulation suite 13CFLUX2 (Weitzel et al., 2013) with extension for INST ${ }^{13} \mathrm{C}-\mathrm{MFA}$ was used according to the workflow described in (Noh et al., 2006) and Suppl. Fig. S2. In brief, cell stage-specific extracellular rates of d0 cells were calculated as described. Mass isotopomer distributions (MIDs) were obtained from two carbon labeling experiments with d0 cells: 1 ) $\left[\mathrm{U}-{ }^{13} \mathrm{C}\right]$ glucose $+\left[{ }^{12} \mathrm{C}\right]$ glutamine and 2) $\left[{ }^{12} \mathrm{C}\right]$ glucose $+\left[\mathrm{U}-{ }^{13} \mathrm{C}\right]$ glutamine. Both data sets were corrected for natural isotope abundance (Niedenfuhr et al., 2016). Data sets were simultaneously inferred with the INST ${ }^{13} \mathrm{C}$-MFA model, justified by the finding that the cell-specific extracellular rates were found very comparable across the three independent biological replicates. In total, 89 unknown parameters (15 net fluxes, 19 exchange fluxes, 31 metabolite pool sizes, 24 groupscale factors) were estimated from 13 cell stage-specific extracellular rate, 15 metabolite pool sizes (averaged over 1) and 2)) and 1002 single timeresolved MID (4-10 time points, 24 metabolites) measurements. Fitting of the unknown model parameters was done by minimizing the variance-weighted least squares function quantifying the difference between the measured and model-predicted measurements. The fitting procedure was repeated 1000 times from random starting points to minimize the risk of getting trapped in local minima. Lastly, for visualization of the best fits the Omix ${ }^{\circledast}$ software was used (Omix Visualization GmbH, Lennestadt, Germany), (Noh et al., 2015).

\subsection{Proteomics analysis}

Cells were grown under normal culture conditions until the desired stage of differentiation. For harvesting, cells were washed with $100 \mathrm{mM}$ Tris-HCl-buffer and harvested by applying urea harvesting solution (8 M urea in $100 \mathrm{mM}$ Tris- $\mathrm{HCl}, \mathrm{pH}$ 8.5). Subsequently, tandem LC/MS 'shot gun' proteomics was performed.

\subsection{Affymetrix gene chip analysis}

Analysis was performed as described earlier (Krug et al., 2013b; Krug et al., 2014). Briefly, samples from approximately $5 \times 10^{6}$ cells were collected using RNA protect reagent from Qiagen. The RNA was quantified using a NanoDrop N-1000 spectrophotometer (NanoDrop, Wilmington, DE, USA), and the integrity of RNA was confirmed with an automated gel electrophoresis system (Experion, Bio-Rad, Hercules, CA, USA). The samples were used for transcriptional profiling when the RNA quality indicator (RQI) number was $>8$. First-strand cDNA was synthesized from $100 \mathrm{ng}$ total RNA using an oligo-dT primer with an attached T7 promoter sequence, followed by the complementary second strand. The double-stranded cDNA molecule was used for in vitro transcription (IVT, standard Affymetrix procedure) using Genechip 30 IVT Express Kit. During synthesis of the aRNA (amplified RNA, also commonly referred to as cRNA), a biotinylated nucleotide analogue was incorporated, which serves as a label for the message. After amplification, aRNA was purified with magnetic beads and $15 \mu \mathrm{g}$ of aRNA was fragmented with fragmentation buffer as per the manufacturer's instructions. Then, $12.5 \mu \mathrm{g}$ fragmented aRNA were hybridized with Affymetrix Human Genome U133 plus 2.0 arrays as per the manufacturer's instructions. The chips were placed in a GeneChip Hybridization Oven- 645 for $16 \mathrm{~h}$ at $60 \mathrm{rpm}$ and $45^{\circ} \mathrm{C}$. For staining and washing, Affymetrix HWS kits were used on a Genechip Fluidics Station-450. For scanning, the Affymetrix Gene-Chip Scanner-3000-7G was used, and the image and quality control assessments were performed with Affymetrix GCOS software. All reagents and instruments were acquired from Affymetrix (Affymetrix, Santa Clara, CA, USA). The generated CEL files were used for further statistical analyses. The authors declare that microarray data were produced according to MIAME guidelines.

\subsection{Transcriptomics data processing and analysis}

The microarray data analysis workflow was assembled using the Konstanz Information Miner (KNIME) open source software (http:// www.knime.com). The raw data was preprocessed using Robust Multiarray Analysis (RMA). Background correction, quantile normalization, and summarization were applied to all expression data samples, using the RMA function from the affy package of Bioconduct (Balmer et al., 2012; Rempel et al., 2015; Shinde et al., 2015). Low-expression genes with a signal below an intensity of 64 in any one of the conditions were filtered out. The limma package ( $\mathrm{R} \&$ Bioconductor) was used to identify differentially expressed genes, with untreated cells set as control group. The moderated t-statistics was applied and was used for assessing the raw significance of differentially expressed genes. Then, final p-values were derived by using the Benjamini-Hochberg method to control the false discovery rate (FDR) due to multiple hypothesis testing. Transcripts with FDR adjusted p-value of $\leq 0.05$ and fold change values $\geq|4|$ were considered significantly regulated. Gene Ontology (GO) analysis for regulated genes were performed using DAVID platform (Huang da et al., 2009b; Huang da et al., 2009a). Transcriptomics data were deposited at GEO database and can be accessed under the number GSE107999.

\subsection{Statistics and data display}

If not otherwise specified, the data shown are the means \pm SD of three independent experiments (each with several technical replicates). 
Independent experiments were performed with different cell batches and they are here termed biological replicates (Schmidt et al., 2017) to distinguish them from technical replicates. Significance calculations are based on ANOVA followed by a suitable post-hoc test (unless otherwise indicated). For data display, the program GraphPad Prism 5 (La Jolla, CA, USA) was used.

\section{Results}

\subsection{Basic omics data for test system characterization}

LUHMES cells have been characterized in their proliferating state (d0) and as differentiated neurons (d6) for expression of characteristic markers (Lotharius et al., 2005; Schildknecht et al., 2009), for the sensitivity to the neurotoxicant $\mathrm{MPP}^{+}$(Poltl et al., 2012) and for electrophysiological properties (Scholz et al., 2011). To get systemswide information on the cells and the main features that distinguish d0 and $\mathrm{d} 6$ cells, we measured gene expression on the whole genome level. Using Affymetrix microarray analysis, we identified about 1400 genes that were $\geq 4$ times higher expressed in one stage compared to the other (Fig. 1A). Overrepresented GO terms were determined separately for genes specific for $\mathrm{d} 0$ and for genes expressed higher on $\mathrm{d} 6$. As expected, GO terms related to cell cycle and proliferation were found for the d0 LUHMES. The GO characteristic for d6 LUHMES were mainly related to the neuronal phenotype (Fig. 1A). Additionally, overrepresented pathways were identified using the Panther and Reactome databases (Thomas et al., 2003; Thomas et al., 2006; Croft et al., 2014; Fabregat et al., 2017). The pathway enrichment corroborated the findings from the GO term analysis (e.g. downregulation of cell cycle pathways and DNA building-block synthesis) and confirmed neuronal differentiation as well as out previous observation that the pathway of amyloid beta production is upregulated in mature LUHMES cells (d6) (Scholz et al., 2013). Amongst the DEG upregulated on d6, no single pathway linked to cellular metabolism was enriched. Thus, the differential gene expression indicates clear differences between the two stages of the cell line on the transcriptome level, but metabolic differences could not be identified from this type of data (Suppl. Fig. S3).

The transcriptomics observations were supported by proteome analysis, which showed a clear difference in the proteome of the two cell differentiation stages with large groups of differentially-abundant proteins in either d0 or d6 cells (Fig. 1B). A substantial fraction of proteins related to oxidative phosphorylation, the TCA and fatty acid metabolism were found to be more abundant in d6 LUHMES. For instance, the proteomics data showed upregulation of the TCA-associated proteins isocitrate dehydrogenase- 1 and 2 (IDH1, IDH2) and of the respiratory chain complex II protein SDHAF2 upon differentiation. Proteins typically more abundant in d0 cells related to the cell cycle and to synthesis of nucleotides (required for DNA synthesis in proliferating cells). Of the pivotal central carbon metabolism (CCM) proteins, malic enzyme 1 (ME-1) was stronger expressed in undifferentiated neurons compared to differentiated ones (data not shown). Malic enzyme (ME) facilitates the channeling of carbon from TCA intermediates into glycolysis to generate pyruvate (gluconeogenesis). Besides added carbon supply of pyruvate, the reaction through ME allows synthesizing NADPH. Within the group of transporters, the strongest upregulations observed upon differentiation were found for the mitochondrial citrate and ATP transporters (SLC25A1/A24).

A typical cellular strategy of metabolic switching involves isoenzyme changes. Examples were observed here for hexokinase (HK) and phosphofructokinase (PFK). The isoenzymes HK2 and PFKP were strongly downregulated in d6 LUHMES, which was compensated by stronger expression of HK1 and PFKM (Fig. 1C). This is consistent with general biochemical knowledge associating HK2 with muscle, adipose tissue, cancer, neural progenitor cells and anabolic metabolism, and HK1 with expression in the brain (Smith, 2000; Wilson, 2003; Zheng et al., 2016). Concordantly, PFKM is known to be predominantly expressed in muscle and brain (Kahn et al., 1979). These findings corroborate on the proteomics level that LUHMES cell differentiate from precursor cells to typical brain tissue parenchymal cells.

The strong alterations in gene transcription and protein expression suggested clear differences in the metabolome of the cells. This was confirmed by metabolomics measurements. The data allowed the clear distinction between different stages of cell differentiation according to their metabolome (Fig. 1D). By a non-targeted metabolomics approach, 38 metabolites were identified and 3 representative metabolites that were downregulated and 6 representatively upregulated metabolites were quantified on the basis of standards. The lower abundance of AMP in differentiated cells is in concordance with the downregulation of purine synthesis genes (Fig. 1B). The higher abundance of glutamine and creatine indicates changes in the CCM and substrate usage while the higher abundancy of D-erythrose may indicate changes in the pentose phosphate pathway.

In summary, this basic characterization of $\mathrm{d} 0$ and $\mathrm{d} 6$ neuronal cell differentiation stages suggests metabolic switches that could affect resilience or susceptibility to toxicants. The suggested metabolic shift, which was not predictable on transcriptome level, but indicated on proteome level, was therefore further investigated.

\subsection{Change of mitochondrial function during differentiation}

To characterize the potential metabolic changes identified by omics approaches in greater detail, mitochondrial physiology was assessed by measurements of the oxygen consumption rate (OCR) using Seahorse technology. A strategy of sequential injection of tool compounds interfering with the mitochondrial respiration was chosen (Fig. 2A). This allowed the calculation of various functional parameters for mitochondria (Fig. 2B). D6 neurons had a slightly higher basal respiration compared to d0 precursor cells, and massive differences became evident, when mitochondria were uncoupled (Fig. 2C). The OCR data showed that the maximal mitochondrial respiration, the spare mitochondrial capacity and the amount of oxygen consumption used for ATP production was significantly increased in d6 cells compared to their d0 precursors (Fig. 2D). Overall, the data suggest that mitochondria run at their maximum in $\mathrm{d} 0$ cells, while $\mathrm{d} 6$ cells have a large spare capacity to increase mitochondrial function according to demand.

\subsection{Change of glycolytic parameters during differentiation of neurons}

To further characterize the central carbon metabolism, glycolysis was investigated for both differentiation stages by measuring extracellular acidification (ECAR; mainly caused by glycolytic lactate secretion). To obtain a broad set of data, ECAR was assessed for different metabolic situations that were provoked by a scheduled injection of tool compounds into cell culture medium (Fig. 3A). D0 and d6 cells differed in their glycolytic capacity (Fig. 3B): proliferative d0 LUHMES cells have a much higher glycolytic rate and capacity in comparison to differentiated d6 neurons. However, d0 cells had no glycolytic reserve to adapt to a higher demand, while d6 cells showed a significant capacity to upregulate glycolysis, when challenged by a mitochondrial inhibitor (Fig. 3B + C).

\subsection{Macroscopic view on the overall metabolic activity}

For further evaluation of the metabolism, the cell numbers of the two cultures were determined. D6 cells were confirmed to be post-mitotic, i.e. their cell number remained constant over $72 \mathrm{~h}$ (Fig. 4A), implying zero growth. In stark contrast, d0 cells proliferated (doubling time about $10-16 \mathrm{~h}$ ) with a specific growth rate of $\mu=0.074 \pm 0.013 \mathrm{~h}^{-1}$ within the first $12 \mathrm{~h}$ of cultivation (SD from at least three biological replicates) (Fig. 4A). After 12-18 h, the $5.5 \mathrm{mM}$ glucose of the labelling medium were exhausted (Fig. 4C). The subsequent metabolic shift resulted in a significant drop of the growth rate 

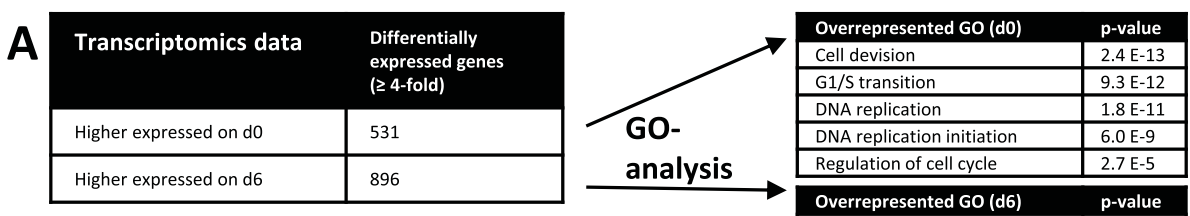

B

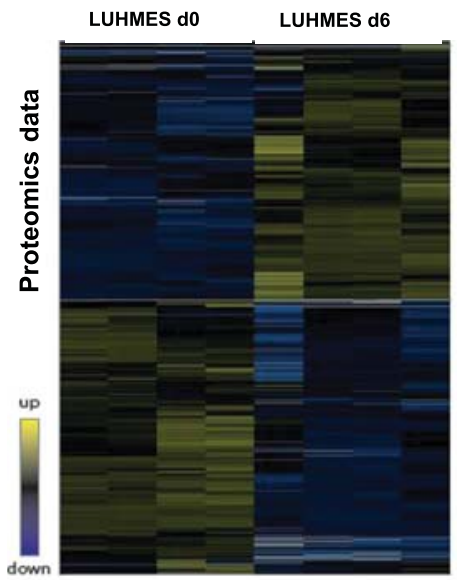

$\frac{\text { Oxidative phosphorylation }}{\text { (43 of } 69 \text { proteins regulated) }}$

TCA cycle

26 proteins regulated) Fatty acid metabolism (15 of 25 proteins regulated)

$\frac{\text { Purine metabolism }}{\text { (42 of } 69 \text { proteins regulated) }}$

Pyrimidine metabolism

(32 of 51 proteins regulated)

Cell cycle

$\frac{\text { Cell cycle }}{\text { (32 of } 52 \text { proteins regulated) }}$

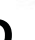

D

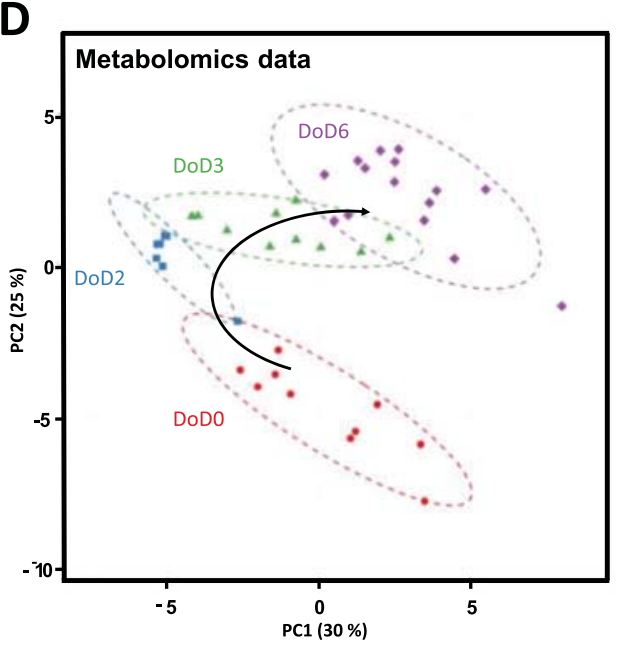

$E$

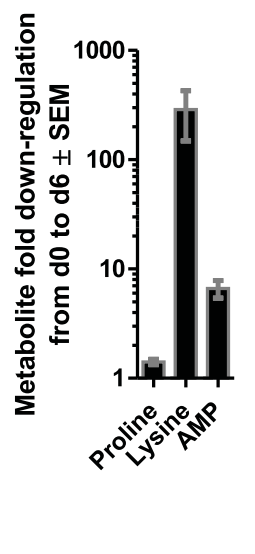

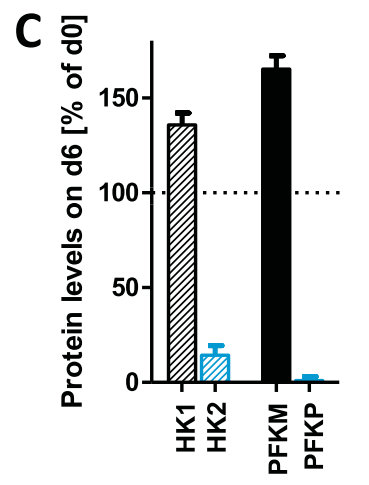

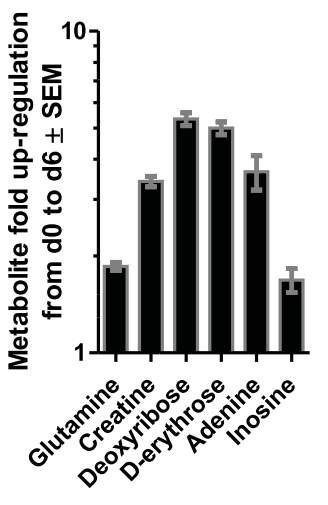

Fig. 1. Changes on transcriptional, proteomic and metabolic level during neuronal differentiation.

For characterization of the proliferating (d0) and differentiated (d6) LUHMES cells, standardized cell culture conditions were used to generate samples from the two types of untreated control cells for further analysis. A: The mRNA expression of four independent cultures of proliferating (d0) and differentiated LUHMES (d6) cells was measured using Affymetrix microarray analysis. Expression levels were determined genome-wide. From these data, differentially expressed genes (DEG) were identified by sorting for probe sets ( $=$ Affymetrix gene probes) that were expressed in $\mathrm{d} 6$ cells $\geq 4$ times higher or lower than in d0 cells. Then, a modulated Limma test was applied to further select those genes for which the differential expression was significant at the $5 \%$ level, after the correction for multiple testing. The number of these genes was then displayed. The DEG were analyzed for statistical overrepresentation of gene ontology (GO) groups using DAVID software. The pvalues indicate the likelihood of a random overrepresentation of the respective GO. The five most-highly overrepresented GO are indicated for the group of genes specific for d0 or d6. B: Four independent cultures of proliferating (d0) and differentiated (d6) LUHMES cells were used for proteome analysis. Differential abundance of proteins is depicted in a heatmap displaying row-wise $\mathrm{z}$ factors of expression levels (blue $=$ lower expression, black $=$ no change, yellow $=$ higher expression). Rows and columns are sorted by similarity after non-supervised clustering. For each major cluster (= proteins dominating in d0 (bottom) or d6 (top)), three typical overrepresented metabolic pathways were selected. The numbers indicate how many different proteins of the total pathway are differentially abundant. C: Two enzymes (each with two isoforms) important for switching metabolic regulation were selected to display the type of changes occurring during LUHMES differentiation. Protein levels were quantified by a shotgun proteomics approach. The protein ratio of $\mathrm{d} 6 / \mathrm{d} 0$ is displayed for hexokinase 1 and 2 (HK1, HK2) and for phosphofructokinase $\mathrm{M}$ and $\mathrm{P}$ (PFKM, PFKP). D: Proliferating (d0) LUHMES cells were differentiated for 6 days. On day of differentiation zero (DoD0, red), two (DoD2, blue), three (DoD3, green) and six (differentiated, DoD6, purple; $=\mathrm{d} 6$ ) the cells were lysed to prepare samples for metabolome analysis. The samples were analyzed by LC-MS, and metabolite peaks with differential abundance were detected. For 38 compounds, unambiguous identification was possible and their changes are displayed as a principal component analysis (PCA). Each point displayed in the PCA map represents one sample analyzed for the respective day of differentiation. The dashed lines indicate the PCA space occupied by samples from each differentiation stage ( $\mathrm{n}=6-15)$. The black arrow suggests how differentiating cells move through the 2D PCA space during differentiation. E: Three metabolites, which were specifically downregulated and six that were specifically upregulated from d0 to $\mathrm{d} 6$ of LUHMES differentiation were selected to display the changes occurring during differentiation. Data are means \pm SEM from 6 to 15 samples. (For interpretation of the references to color in this figure legend, the reader is referred to the web version of this article.)

(Fig. 4A) and decreased lactate production (Fig. 4D). Thus, the time regime for INST ${ }^{13} \mathrm{C}$-MFA was limited to $0-12 \mathrm{~h}$, to make sure that cells were still in non-limiting culture conditons. Normalized to their protein content, both cell differentiation stages, d0 and d6, were found to contain the same amount of ATP (Fig. 4B). Absolute cell-specific extracellular rates for both cell differentiation stages are summarized in Suppl. Table 2. These rates provide a means to compare the associated metabolic phenotypes. In concordance with the mitochondrial and glycolytic physiology parameters determined above (Figs. 2 and 3), d0 cells took up glucose much faster from the medium than $\mathrm{d} 6$ cells: glucose uptake rates were $220 \pm 44$ and $109 \pm 13 \mathrm{nmol} / 10^{6} \mathrm{cells} / \mathrm{h}$ for $\mathrm{d} 0$ and $\mathrm{d} 6$, respectively. In case of $\mathrm{d} 0$, the increased glycolytic turnover was paralleled with high uptake and secretion of certain amino acids into the medium. In particular, serine and isoleucine were consumed much faster by $\mathrm{d} 0$ than by $\mathrm{d} 6$ cells. Moreover, proline and alanine were secreted at 3.6-4.9 fold higher rates by proliferative cells $(8 \pm 4$ vs. $2 \pm 1 \mathrm{nmol} / 10^{6} \mathrm{cells} / \mathrm{h}$ and $68 \pm 8 \mathrm{vs} .19 \pm 1 \mathrm{nmol} / 10^{6} \mathrm{cells} / \mathrm{h}$ for proline and alanine in $\mathrm{d} 0$ and $\mathrm{d} 6$, respectively) (Fig. 4E-H, Suppl.
Table 2). Both, d0 and d6 cells experienced high relative lactate secretion rates ( $232 \mathrm{~mol}-\%$ vs. $282 \mathrm{~mol}-\%$ related to glucose uptake rates, respectively) which again underlines high glycolytic activity in both cell stages. Expectedly, on an absolute level d0 cells released more lactate into the medium compared to d6 cells $(509 \pm 56$ vs. $307 \pm 24 \mathrm{nmol} / 10^{6}$ cells $/ \mathrm{h}$, respectively) (Fig. $4 \mathrm{C}+\mathrm{D}$ ).

These findings are well in line with the different metabolic situations of proliferative and adult neuronal cells (Fig. 4I). In post-mitotic d6 neurons, metabolism mainly serves for energy (ATP) production to maintain the functionality of the cell. For this purpose, glucose is metabolized to the level of pyruvate through glycolysis, and likely to $\mathrm{CO}_{2}$ (experimentally not accessible) in subsequent steps in the TCA. The proliferating do cells can also use this pathway, but their major objective is production of biomass. To use their full metabolic capacity, they also take up amino acids (Fig. 4E + F), in particular glutamine, at much higher rates than d6 cells $\left(78 \pm 10\right.$ vs. $6 \pm 3 \mathrm{nmol} / 10^{6}$ cells $/ \mathrm{h}$, respectively) (Suppl. Table 2) (Birsoy et al., 2015; Markert and Vazquez, 2015; Sullivan et al., 2015; Fernandez-de-Cossio-Diaz and 
A

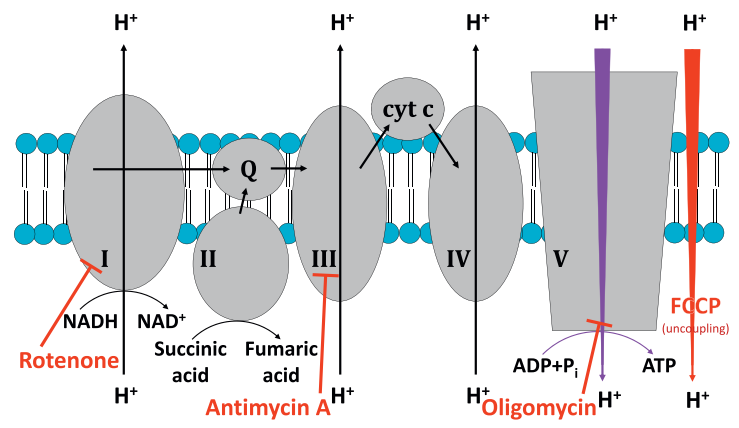

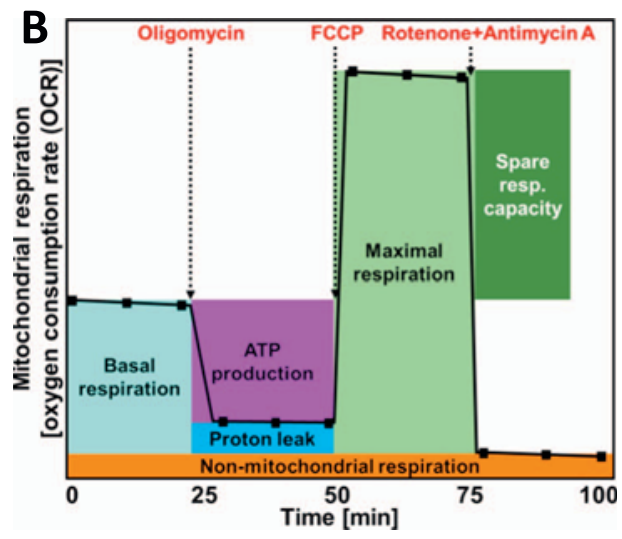

D

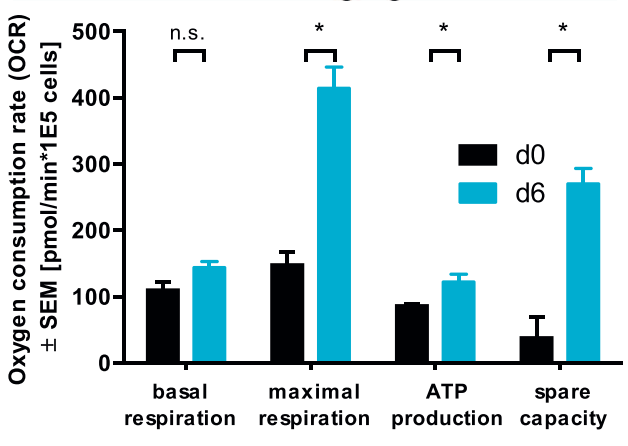

Fig. 2. Differences in mitochondrial function between d0 and d6 LUHMES cells.

A: Scheme of the mitochondrial electron transport chain, with a display of complexes I-V on the inner mitochondrial membrane. Sites of electron and proton transport and their flow are displayed in black, ATP generation driven by the proton-motive force is shown in purple. Targets of inhibitors used for functional analysis are indicated in red. The uncoupling agent FCCP was used to disrupt the proton gradient across the inner mitochondrial membrane. B: Exemplary time course (black curve) of mitochondrial oxygen consumption rate (OCR) under the influence of added inhibitors used by the mitostress test. The scheme displays the standardized schedule of inhibitor injection (time axis). Moreover it indicates how various parameters of mitochondrial physiology are calculated from the OCR of the four experimental phases. C: Proliferating (d0, black) and differentiated (d6, blue) LUHMES cells were analyzed for their mitochondrial functionality using the Seahorse mitostress test. OCR was normalized to the cell number, and the injection of the mitochondrial inhibitors/uncoupler is indicated in red. D: The data from $\mathrm{C}$ were used to calculate mitochondrial physiology parameters as indicated in $\mathrm{B}$. All data are means \pm SEM from two-three biological replicates. *: $\mathrm{p}<0.05$, by one-way ANOVA followed by Dunnett's post-hoc test. (For interpretation of the references to color in this figure legend, the reader is referred to the web version of this article.)

Vazquez, 2017). In addition, glycolysis is used in d0 cells in part to provide building blocks like serine or glycine, which are essential for both protein and nucleic acid (purine and pyrimidine) synthesis. If glutamine, serine and isoleucine are used by cells to generate energy or to build up biomass (including lipids), then cells have to get rid of the excess nitrogen. The need to dispose nitrogen equivalents thus may explain the secretion of alanine (Fig. $4 \mathrm{H}$ ), ornithine (data not shown) and ammonia (Suppl. Table 2) by d0 cells. If glycolysis accounts for a large portion of cellular ATP production, as in do cells, then large amounts of reduction equivalents (NADH) are generated besides pyruvate. Conversion of pyruvate to lactate and subsequent secretion of lactate is one way to maintain this type of metabolism. Similarly, proline can be generated from glutamine/glutamate (Fig. 4G) as a way for cells to get rid of reduction equivalents, when glutamine is used as energy source (Fig. 4I) (Fernandez-de-Cossio-Diaz and Vazquez, 2017).

\subsection{Direct insights from metabolic pool sizes and isotopic labeling incorporation profiles}

To unravel the intracellular metabolic situation, we used the pulsed stable isotope-resolved metabolomics (pSIRM) approach. For this purpose, parallel cultures of cells were exposed to either $\left[\mathrm{U}^{13} \mathrm{C}\right]$ glucose or $\left[\mathrm{U}-{ }^{13} \mathrm{C}\right.$ ] glutamine label (all carbon atoms ${ }^{13} \mathrm{C}$ labeled). At defined times, cellular metabolites were extracted and measured by GC-MS. The resulting data delivered twofold information: first, the overall pool sizes (metabolite amount per cell) of CCM metabolites; second, the label incorporation dynamics into CCM metabolites. Both were used for direct interpretation as well as later on for INST ${ }^{13} \mathrm{C}-\mathrm{MFA}$. The pool sizes were used for metabolic flux determination by INST ${ }^{13} \mathrm{C}$-MFA.
For most intracellular metabolites measured, we found that their pool sizes (concentrations) were within the same order of magnitude in $\mathrm{d} 0$ and $\mathrm{d} 6$ cells; for some glycolysis intermediates and amino acids, d6 cells had higher pool sizes, but the difference was not statistically significant (Fig. 5A). Overall, pool sizes spanned several orders of magnitude with glutamate being the largest pool amounting to 45.3 and $82.4 \mathrm{nmol} / 10^{6}$ cells in $\mathrm{d} 0$ and $\mathrm{d} 6$ cells, respectively. Aspartate was below the detection limit in $\mathrm{d} 6$ cells, while sizable amounts were measured in $\mathrm{d} 0$ precursors. The reverse situation was observed for glutamine. This may be explained by the fact that $\mathrm{d} 0$ cells need to maintain a sizable pool size of aspartate for biomass generation which they derive from cellular metabolism. Furthermore, it could be expected that $\mathrm{d} 0$ cells rapidly convert all glutamine to glutamate which is, in turn, in rapid equilibrium with $\alpha$-ketoglutarate (aKG) feeding into the TCA.

Following the path of glycolytic carbon revealed that incorporation of labeled carbon from $\left[\mathrm{U}^{13} \mathrm{C}\right]$ glucose into the cellular metabolite pools followed the expected order (Fig. 5B): the 3-phosphoglycerate (3PG) pool was $>80 \%$ labeled after $1 \mathrm{~h}$ (as determined by the $[1,2]$ fragment as proxy). This means that most 3-PG present in all cells at that time point was derived from freshly added (labeled) glucose. For lactate and alanine it already took $6 \mathrm{~h}$ to reach $60 \%$ label incorporation, and labeling dynamics of citrate was found to be even slower.

Assuming clockwise (oxidative) operation of the TCA, labeled citrate may be formed from the reaction of non-labeled oxaloacetate (derived from unlabeled glutamate channeled into the TCA via aKG) and $\mathrm{m}+3$ labeled oxaloacetate (derived from $\left[\mathrm{U}-{ }^{13} \mathrm{C}\right]$ glucose via pyruvate carboxylase) with $\mathrm{m}+2$ labeled (mitochondrial) acetyl-CoA (derived from fully-labeled pyruvate). This theoretically yields $\mathrm{m}+2$, 

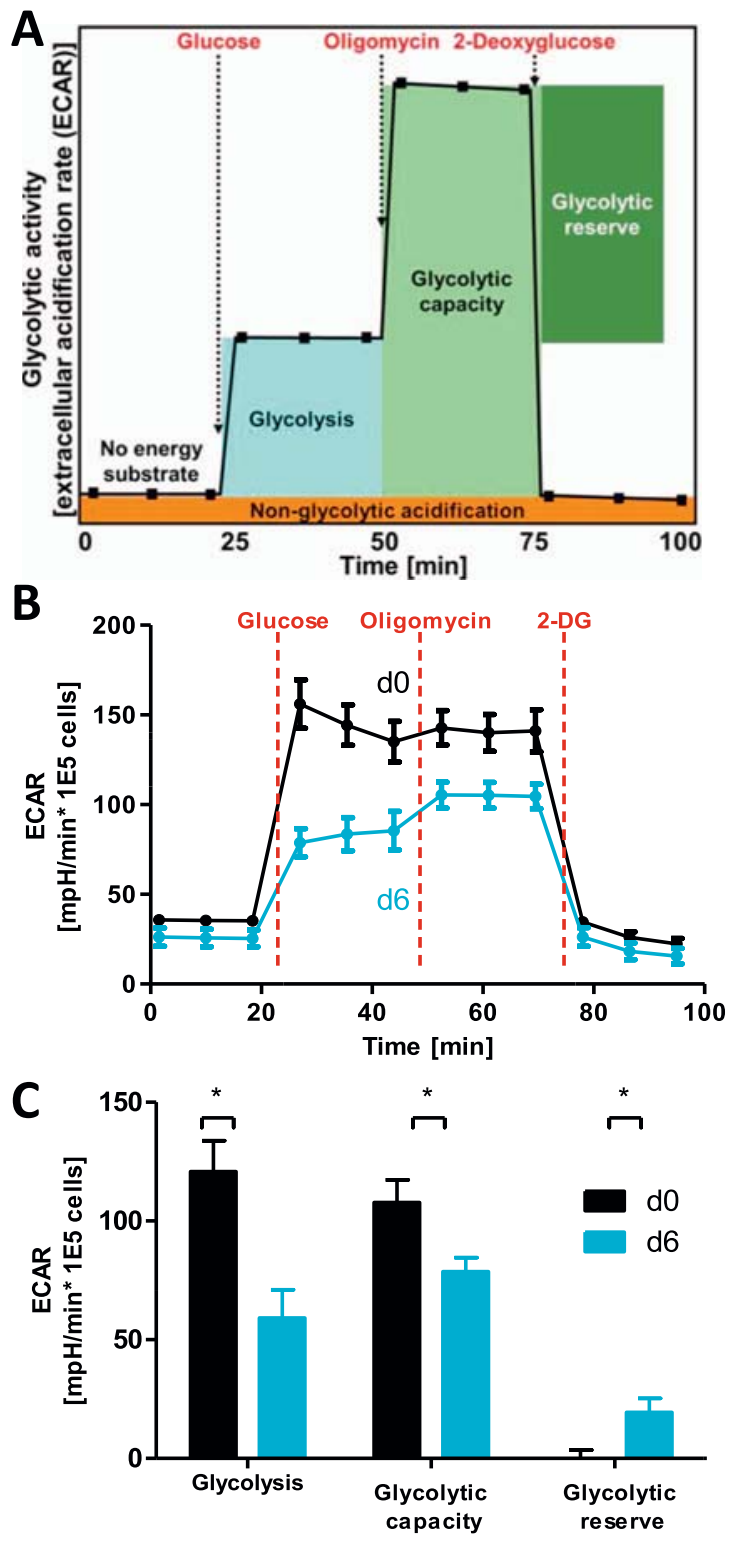

Fig. 3. Differences in glycolytic activity during neuronal differentiation.

A: Exemplary time course (black curve) of extracellular acidification rate (ECAR) under the influence of added glucose and of inhibitors used by the glycostress test. The scheme displays the standardized schedule of inhibitor injection (time axis). Moreover, it indicates how various parameters of glycolytic physiology are calculated from the ECAR of the four experimental phases. B: Proliferating (d0, black) and differentiated (d6, blue) LUHMES cells were analyzed for their glycolytic functionality using the Seahorse glycostress test. ECAR was normalized to the cell number, and the injection of glucose and inhibitors is indicated in red. C: The data from B were used to calculate glycolytic parameters as indicated in A. All data are means \pm SEM from two-three biological replicates. *: $\mathrm{p}<0.05$, by one-way ANOVA followed by Dunnett's post-hoc test. (For interpretation of the references to color in this figure legend, the reader is referred to the web version of this article.)

and $m+5$ labeled citrate while $m+0$ citrate is also the result of the continuous inflow of unlabeled glutamate into the TCA, thereby limiting the labeling fraction of the higher mass traces of citrate. In contrast to these theoretical considerations, only a fragment of citrate could be observed within our analytical setup, i.e., the internal carboxylic acid group is lost during analytical analysis (Suppl. Fig. S1F). This shifts the theoretical considerations above towards the in reality observed $\mathrm{m}$ $+0, m+2$ and $m+4$ traces as shown in Fig. 5B. Although this qualitatively exemplifies the flow of labeled carbons through the metabolic pathways (Fig. 5B), the considerations neglect important aspects of the metabolic network such as malic enzyme, the potential influx of lipids via AcCoA or the label exchange from and to amino acids, e.g. between oxaloacetate and aspartate. To resolve these fluxes, a computational approach is needed (see next section).

The comparison of ${ }^{13} \mathrm{C}$ label incorporation profiles in $\mathrm{d} 0$ and $\mathrm{d} 6$ cells revealed differences in label incorporation speed depending on whether $\left[\mathrm{U}-{ }^{13} \mathrm{C}\right]$ glucose or $\left[\mathrm{U}-{ }^{13} \mathrm{C}\right]$ glutamine tracers were administered (Fig. 5C, Suppl. Fig. 1). For $\left[\mathrm{U}^{13} \mathrm{C}\right]$ glucose we found that d0 cells produced lactate faster compared to d6 cells (Fig. 5C and Suppl. Fig. S1A, C), which is in line with the data generated by the Seahorse measurements indicating a higher lactate efflux in d0 cells (Fig. 3C). Furthermore, label incorporation dynamics in the malate pool suggests that malate was turned over faster in $\mathrm{d} 6$ cells while the turnover of citrate was slightly slower in d6 (Fig. S1, C). This might point towards pronounced channeling of carbon, derived from glucose, or further species, such as extracellular pyruvate, into mitochondria of mature cells via TCA and/or anaplerotic reactions compared to d0 cells (Fig. 5C). However, labeling data of citrate was only obtained for a short labeling time $(6 \mathrm{~h})$, and the situation would need to be clarified for longer labeling periods $(24 \mathrm{~h})$.

Feeding $\left[\mathrm{U}_{-}{ }^{13} \mathrm{C}\right]$ glutamine to both cell stages and following the incorporation of labeled ${ }^{13} \mathrm{C}$ into metabolites of the TCA gave additional information: for both citrate and malate the fraction of labeled metabolite increased much faster in d0 than in d6 cells (Fig. 5C, Fig. $\mathrm{S} 1 \mathrm{~B}, \mathrm{D})$. Thus, glutaminolysis is significantly enhanced in d0 compared to d6 cells which is in line with extracellular rate measurements. Since the central carbon metabolism can also potentially operate in glyconeogenetic direction (Nicolae et al., 2014), we also studied whether glutamine was converted to lactate. The labeling fraction of intracellular lactate in cells exposed to $\left[\mathrm{U}_{-}{ }^{13} \mathrm{C}\right]$ glutamine informed about a potential channeling of TCA metabolites back into glycolysis (Fig. S1E). Here, only a minor degree of label incorporation was observed in lactate compared to TCA metabolites (Fig. S1D). There was also no difference between d0 and $\mathrm{d} 6$ cells, although ME was expressed stronger in d0 cells (Fig. 1). This data provides evidence that LUHMES cells do not use glutamine to generate glycolysis metabolites (Suppl. Fig. S1). To approach the metabolic activities in LUHMES cells, knowledge of the actual intracellular metabolic carbon fluxes is required.

\subsection{Metabolic flux analysis of do neuronal precursors}

While time-resolved label incorporation dynamics gives qualitative insights into the metabolic operation modes of LUHMES cells, the quantification of intracellular fluxes requires a model-based approach. INST ${ }^{13} \mathrm{C}$-MFA integrates measurements of extracellular rates, metabolite pool sizes and CCM labeling incorporation measurements into a metabolic network model to jointly infer metabolic fluxes and pool sizes on the basis of mass balances. The final result of this analysis is a flux and pool size distribution giving absolute numbers for all parameters of the ${ }^{13} \mathrm{C}$ flux model. From this distribution, a so called flux map is generated that shows the calculated fluxes and pool size distributions in the context of the metabolic network. In this study, we applied INST ${ }^{13} \mathrm{C}$-MFA to derive the flux map of $\mathrm{d} 0$ cells. The computational process is overviewed in Suppl. Fig. S2. Best solutions for all reaction parameters of the metabolic model were determined in an iterative parameter fitting procedure by minimization of the deviation between the model-predicted values and real measurements. Good fits are obtained for all but one extracellular rate (i.e. influx into mitochondrial acetylCoA), all MIDs and pool sizes. The flux map of the best fit is shown in Fig. 6 with fluxes expressed in absolute numbers ([nmol/h/10 $/ 10^{6}$ cells $]$ ). Flux values in absolute numbers as well as in \% of the glucose uptake rate are found in Suppl. Table 3.

A major metabolic feature immediately evident from the do flux map is the predominant glucose to lactate metabolism (Fig. 6A). Experimentally determined cell-number specific extracellular fluxes, 

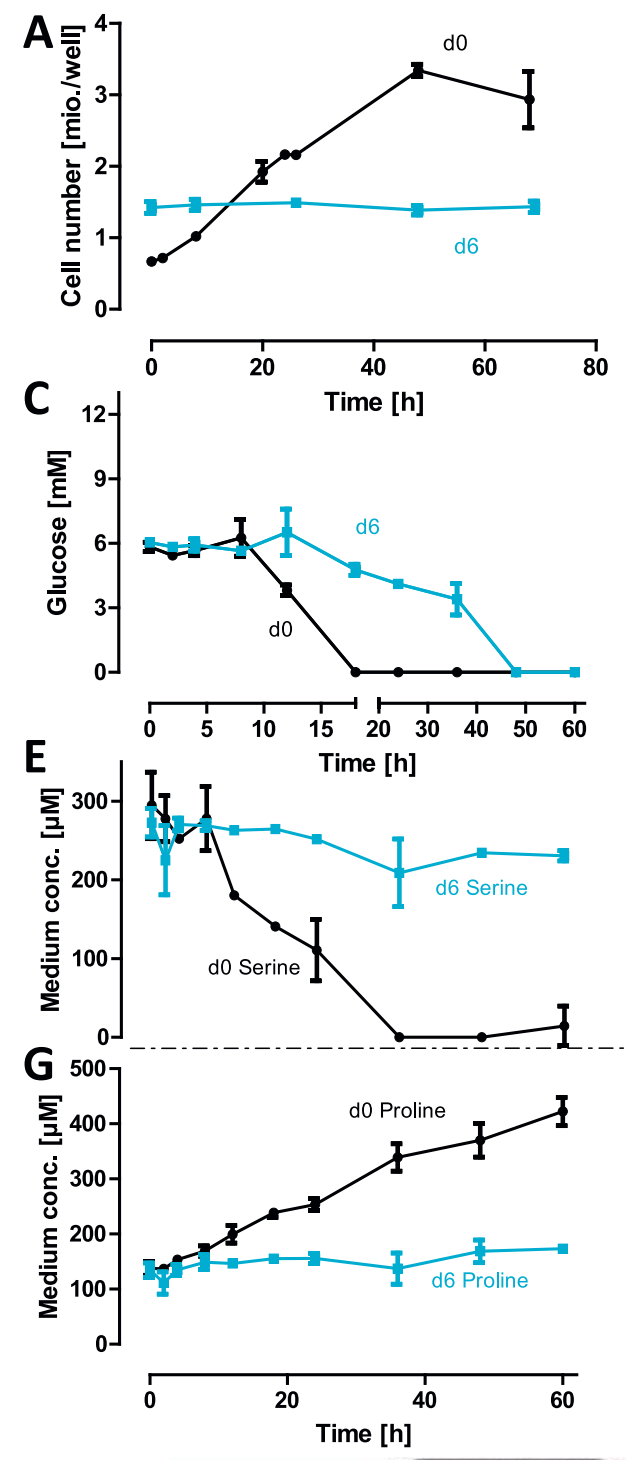
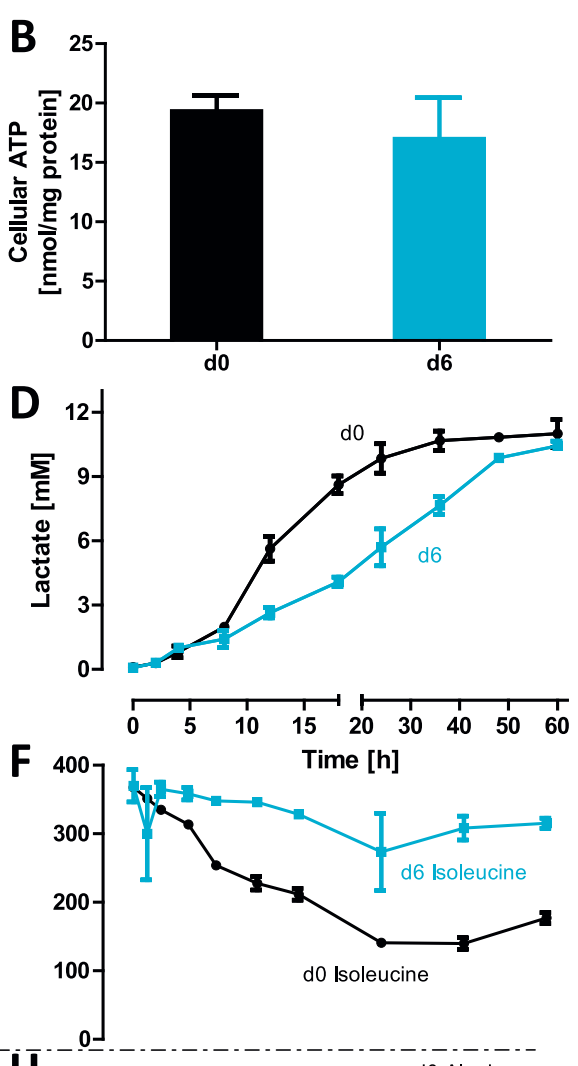

H

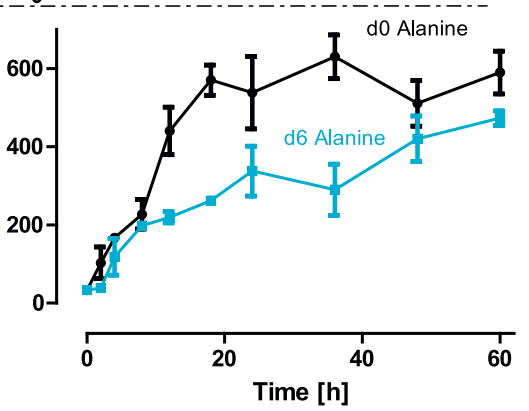

Fig. 4. Change of substrate utilization during neuronal differentiation.

LUHMES cells (d0 or d6) were cultured under standardized conditions. Various culture parameters were analyzed for up to $72 \mathrm{~h}$, i.e. d0 cells (black) were allowed to continue proliferation for up to three days, and d6 cells (blue) remained in the state of differentiated neurons for up to $72 \mathrm{~h}$. Data are means \pm SD from three independent experiments. A: Change in cell number (depicted in million cells per well) for d0 and d6 LUHMES over $72 \mathrm{~h}$. B: ATP levels of d0 and d6 LUHMES. Data are normalized to cellular protein content, which was determined in parallel. C + D: Pool sizes (concentrations) of glucose (C) and lactate (D) in the cell culture medium. Measurements were performed for up to $60 \mathrm{~h}$ in cultures of d0 and d6 cells. E-H: Pool sizes (concentrations) of serine (E), isoleucine $(\mathrm{F})$, proline $(\mathrm{G})$ and alanine $(\mathrm{H})$ in the cell culture medium. Measurements were performed for up to $60 \mathrm{~h}$ in cultures of $\mathrm{d} 0$ and $\mathrm{d} 6$ cells. I: Schematic illustration of differences in metabolism between $\mathrm{d} 0 / \mathrm{d} 6$ cells. The metabolism of post-mitotic $\mathrm{d} 6$ neurons mainly serves the energy production (ATP) to maintain the cells functional. For this purpose, glucose is metabolized to the level of $\mathrm{CO}_{2}$ through glycolysis and subsequent steps in the tricarboxylic acid cycle (TCA) and respiratory chain. The proliferating do cells can use similar pathways, but their major objective is production of biomass (proliferation). Therefore, they take up amino acids, in particular glutamine being a building block for proteins and a nitrogen carrier for nucleotide synthesis. Also glycolysis is used in part to provide building blocks like serine or lysine. Aspartate is exemplified as key building block that can be imported and/or assembled from multiple sources, and that is essential for both protein and nucleic acid (purine and pyrimidine) synthesis. Secretion of ornithine, alanine or urea/ammonia serves as nitrogen sink. If glycolysis is used for energy production, as in $\mathrm{d} 0$ cells, then excess reducing equivalents need to be disposed of in mitochondria (electron sink). Alternatively, this can be achieved by disposal of lactate, or by generation of proline from glutamate (when glutamine is used as energy source). (For interpretation of the references to color in this figure legend, the reader is referred to the web version of this article.)

I

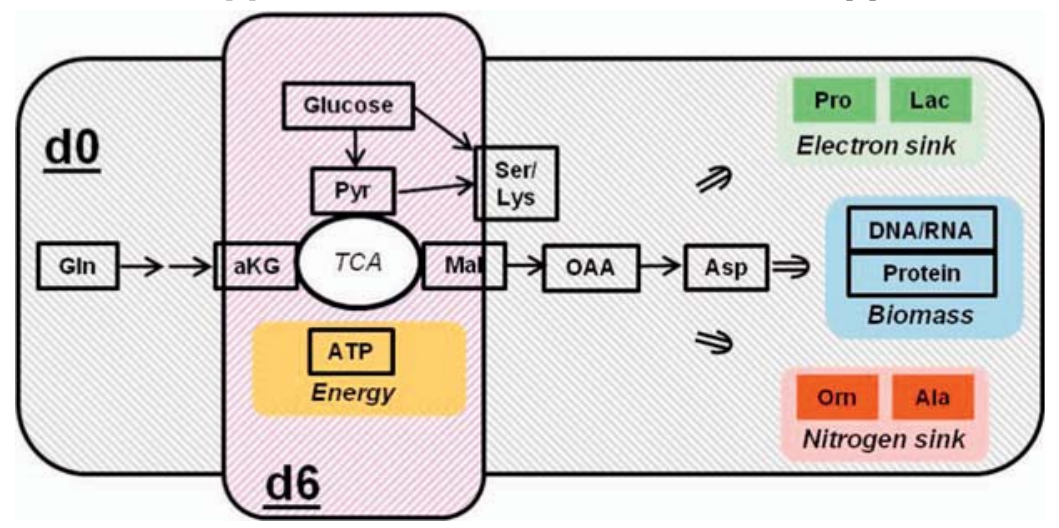

particularly glucose uptake, and the dominant lactate efflux, are well reproduced by the model (Fig. 6B, E). Also extracellular uptake and secretion rates of amino acids coincide well, such as aspartate and glutamine uptake rates (Fig. $6 \mathrm{C}$ ), as well as alanine and proline secretion rates (Fig. 6E). Additional carbon influx into the CCM occurred through mitochondrial acetyl-CoA and succinate as well as the glutamate pool due to catabolism of extracellular amino acids (Fig. 6C) and metabolic requirements for biomass synthesis. It should be noted that in the model threonine, leucine, isoleucine, phenylalanine, tyrosine, tryptophan and lysine entered the CCM via mitochondrial acetyl-CoA, valine, methionine, isoleucine, phenylalanine and tyrosine via succinate as well as histidine and arginine via glutamate.

INST ${ }^{13} \mathrm{C}$-MFA provides detailed insight into glycolytic as well as glutaminolytic intracellular fluxes into the TCA. Fluxes (in \% of glucose uptake) (Fig. 6D) suggest that the TCA is approximately equally fueled through glycolysis and glutaminolysis. While the net flux through citrate synthase is $25 \%$, the net flux from glutamate to aKG (involving glutamate dehydrogenase) is $18 \%$. As a consequence, the succinate dehydrogenase flux from succinate towards fumarate within the TCA is $42 \%$. The results stress the important role of glutaminolysis, on the one 

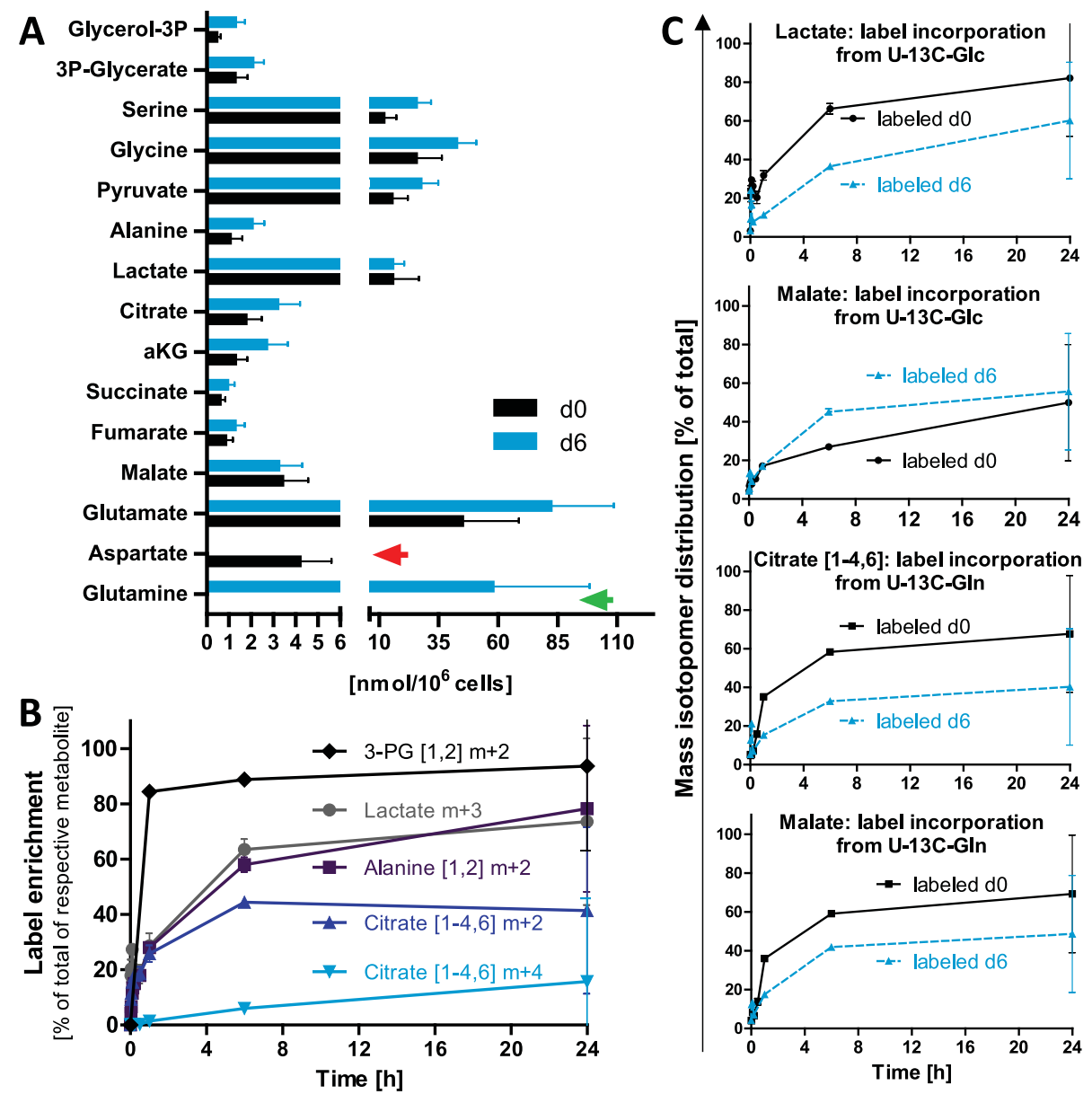

Fig. 5. Intracellular metabolite pool sizes and label incorporation.

LUHMES cells (d0 or d6) were cultured under standardized conditions, until medium was replaced with fresh media $4 \mathrm{~h}$ prior to label experiments. At $0 \mathrm{~h}$, medium was replaced again with culture medium either supplemented with $\left[\mathrm{U}_{-}-{ }^{13} \mathrm{C}\right]$ glucose $+\left[\mathrm{U}_{-}{ }^{12} \mathrm{C}\right]$ glutamine or $\left[\mathrm{U}-{ }^{12} \mathrm{C}\right]$ glucose $+\left[\mathrm{U}_{-}{ }^{13} \mathrm{C}\right]$ glutamine. Various culture parameters, such as intracellular metabolite pool sizes and label incorporation into metabolites were analyzed for up to $24 \mathrm{~h}$. For 3-PG, alanine and citrate only the [1,2], [1,2] and [1-4,6] fragments, respectively, were observed (InChI enumeration). A: Comparison of intracellular central carbon metabolism (CCM) metabolites of d0 and d6 LUHMES. The red arrow highlights the aspartate pool, only identified in do cells; the green arrow indicates the glutamine pool, only identified in d6 neurons. B: Label incorporation into CCM metabolites in d0 LUHMES after the exposure to $\left[\mathrm{U}^{-13} \mathrm{C}\right]$ glucose. The number of incorporated heavy atoms is indicated (e.g. $\mathrm{m}+2$ means two ${ }^{13} \mathrm{C}$ atoms instead of ${ }^{12} \mathrm{C}$ atoms). C: Time-resolved label incorporation over $24 \mathrm{~h}$ into CCM metabolites from $\left[\mathrm{U}^{-1}{ }^{13} \mathrm{C}\right]$ glucose or $\left[\mathrm{U}-{ }^{13} \mathrm{C}\right]$ glutamine, as indicated. For better visualization, the summarized percentage of all label containing fragments is displayed (y-axis). All data are means \pm SD from three independent experiments. (For interpretation of the references to color in this figure legend, the reader is referred to the web version of this article.) hand, to fuel the TCA and, on the other hand, to accomplish the required demand for biomass synthesis, e.g. for fatty acid synthesis via acetyl-CoA. The flux from the cytosolic acetyl-CoA pool into the biomass was $10 \%$ of the glucose uptake, while the flux from mitochondrial acetyl-CoA pool into biomass was approximately found to be twice as high (19\%) (Fig. 6A, Suppl. Table 2). The overall flux into the biomass was determined to be $139.7 \mathrm{nmol} / 10^{6}$ cells/h (or $405 \mathrm{C}-\mathrm{nmol} / 10^{6}$ cells/ h, i.e. number of carbon atoms), representing $53 \%$ of the glucose uptake. Thus, the high flux into the biomass in $\mathrm{d} 0$ underlines the need of biomass precursors to accomplish cellular proliferation expressed by the high specific growth rate compared to zero growth in d6 $\left(\mu=0.074 \mathrm{~h}^{-1}\right.$ vs. $\left.0.0 \mathrm{~h}^{-1}\right)$.

In d0 cells we observed strict clockwise (oxidative) operation of the TCA, potentially to enable the cells to meet their NADH and ATP requirements. In particular, no reversible carboxylation of aKG to citrate occurs ( $<10 \mathrm{e}^{-6} \mathrm{nmol} / 10^{6}$ cells $/ \mathrm{h}$ ). Interestingly, this is in contrast to the findings reported by Sá et al. (Sa et al., 2017) for murine neural stem cells. Notably, by integrating all available measurements in the network model, fluxes entering the TCA via pyruvate dehydrogenase (PDH, lower glycolysis) and pyruvate carboxylase (PC, anaplerosis) can be destinguished by ${ }^{13} \mathrm{C}$-MFA. A high PDH-flux of $32 \%$ was estimated by ${ }^{13} \mathrm{C}-\mathrm{MFA}$. This would satisfy the demands for acetyl-CoA precursors for biomass generation and fuel the TCA. The PC-flux contributed only to a minor extent (ca. 5\%). From these flux estimates, a PDH/PC ratio of ca. 0.15 is derived for d0 cells, which is only slightly lower than the ratio of 0.18 determined for neural stem cells by Sá et al. (Sa et al., 2017).

Assuming that intracellular serine is exclusively used for purine synthesis, an upper bound for the purine synthesis flux can be derived from the total flux into the serine pool. This gives a maximal purine synthesis flux of ca. $7 \%$ of the glucose uptake $(2.5 \%$ and $4.5 \%$ derived from the uptake of glucose and serine, respectively), which is in-line with the low carbon fluxes through the pentose phosphate pathway (Suppl. Table 3).

Summarizing, a model-based representation of the CCM network of d0 LUHMES cells was has been established explaining specific extracellular rates, metabolic pool sizes and labeling incorporation dynamics in a consistent manner. The ensuing flux map showed a metabolism that is focused on aerobic glycolysis, but also involved the TCA and amino acid metabolism to a substantial extent. The same MFA approach, as applied for $\mathrm{d} 0$ cells was attempted for $\mathrm{d} 6$ cells. In this case we found that the quality standards for quantitative flux modeling could not be met (Suppl. Fig. S2). Here, future research work is required to collect data in the quality needed by mathematical modeling to allow the assembly of highly resolved flux maps for complex mammalian cells and in particular to answer the question whether the altered mitochondrial physiology parameters in $\mathrm{d} 6$ cells are reflected by respective changes in metabolic fluxes.

\subsection{Loss of glutamine dependency upon differentiation}

Further experiments were conducted to investigate the impact of media components such as pyruvate and glutamine on cell growth. The data showed that not only glutamine, but also glutamate and aspartate were rapidly taken up by do cells from the medium, while d6 cells reduced the medium composition of these amino acids only marginally (Fig. 7A). To follow up on this, we examined to which extend the cells required glutamine supplementation. The d6 cells survived for $24 \mathrm{~h}$ in medium devoid of glutamine and pyruvate, while do cells all died. Glutamine was able to keep cells surviving, but it did not allow 


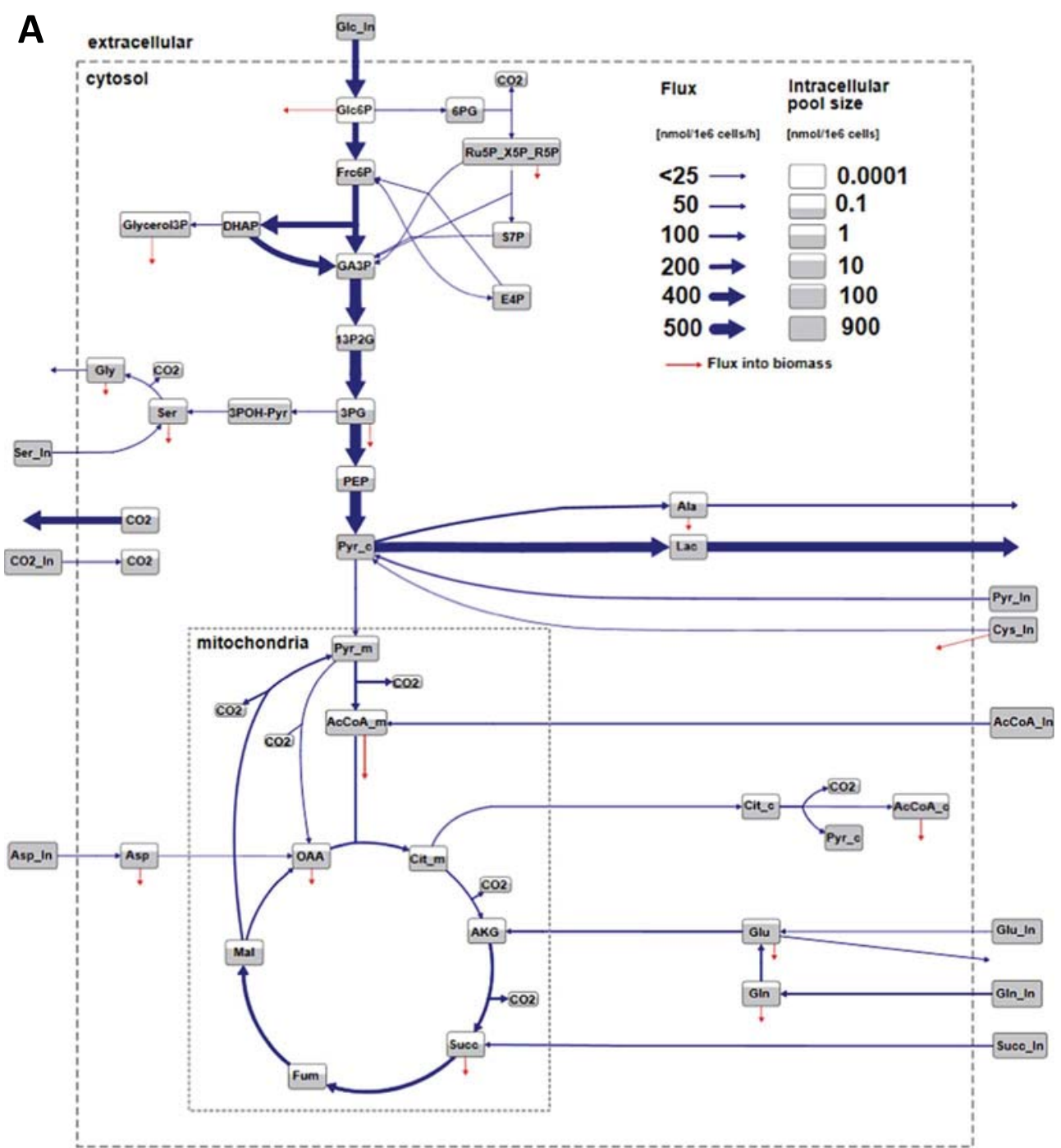

Fig. 6. Flux map of proliferating LUHMES cells. For proliferating LUHMES cells, metabolic flux analysis (MFA) was performed according to Suppl. Fig. S2, and the data are summarized in a metabolic flux map. A: The CCM-fluxmap features glycolysis, the TCA, as well as input and output fluxes of metabolites. Flux velocity was encoded by the arrow size, the metabolite pool size (= concentration) was encoded by the amount of background coloring in the text boxes. Fluxes into biomass are depicted as red arrows pointing to no specific target. The model assumes three compartments (extracellular space, cytosol and mitochondria). A list of abbreviations regarding metabolic pools used for the flux map can be found in Suppl. Table $1 \mathrm{~B}$, C: Measured fluxes were compared to simulated fluxes derived from the MFA flux model. The net input fluxes into glycolysis (B), and into the TCA (C) are displayed. Additional relative information on the metabolic routes considered is added to the data displays. D: Selected intracellular fluxes, relative to glucose uptake (AcCoA_c $=$ cytosolic acetyl-CoA, $\mathrm{BM}=$ biomass). $\mathrm{E}$ : Measured secretion rates (e.g. lactate secretion) are displayed together with data derived on the same fluxes from MFA modeling as shown in A. (For interpretation of the references to color in this figure legend, the reader is referred to the web version of this article.)
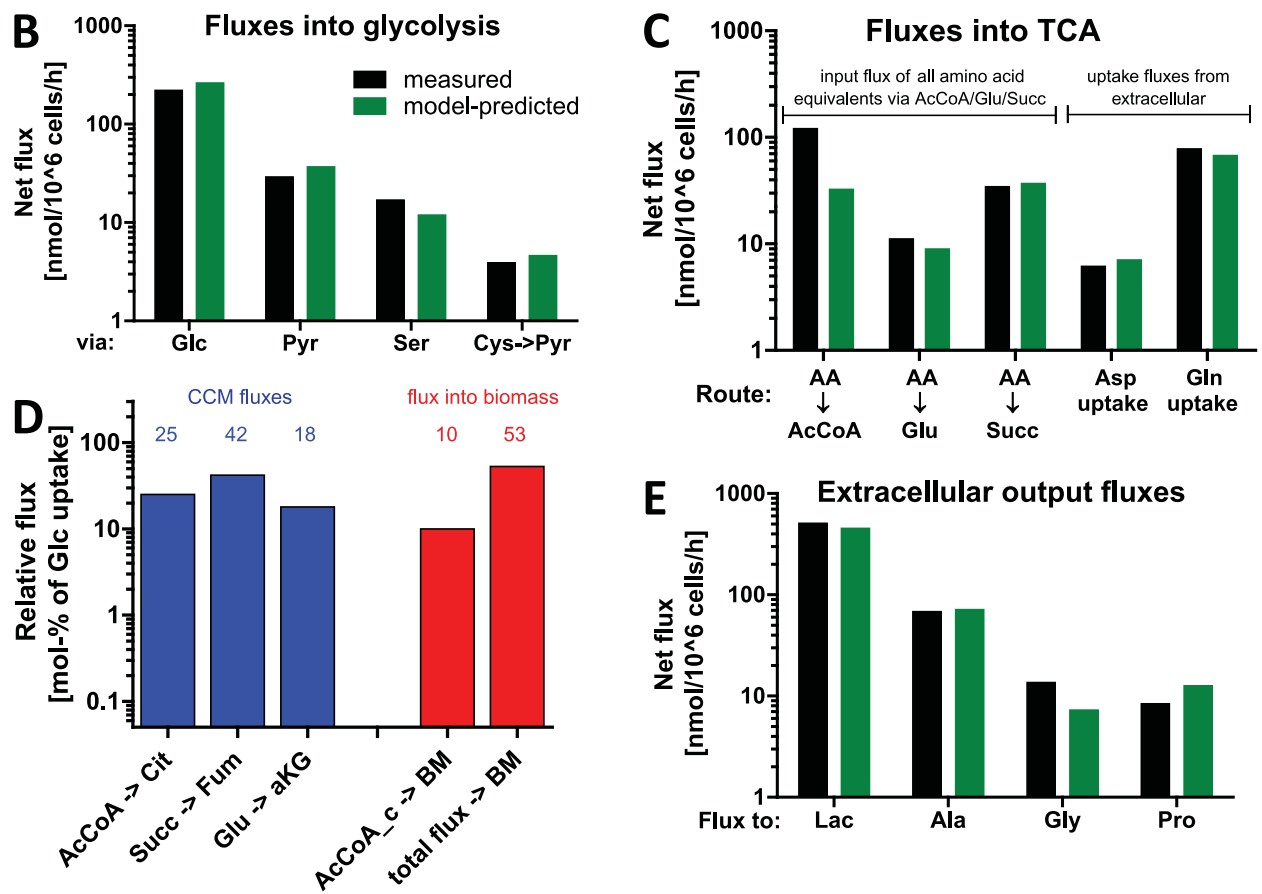


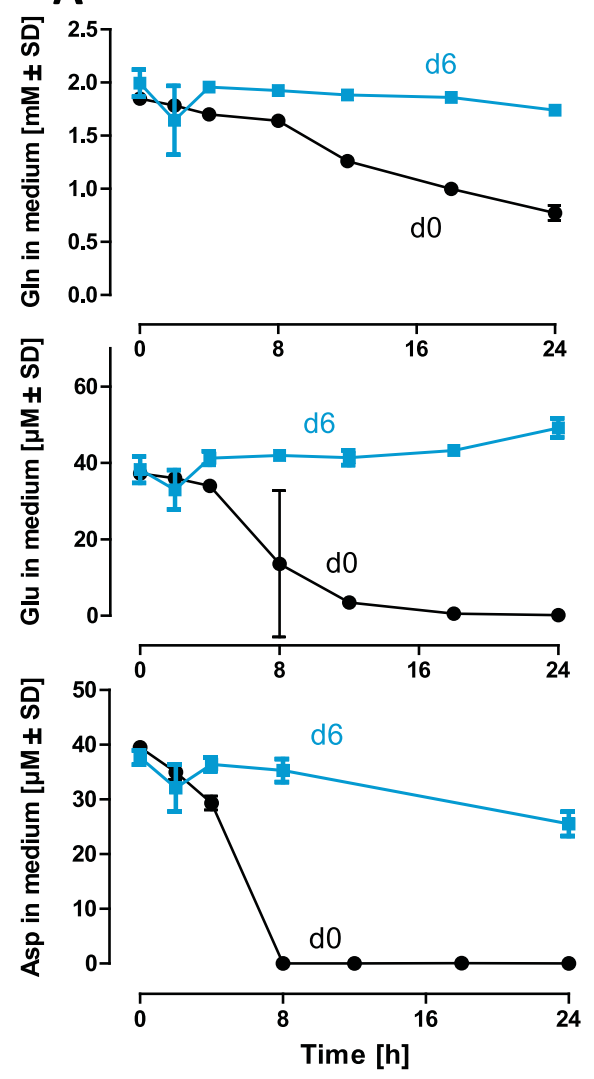

B
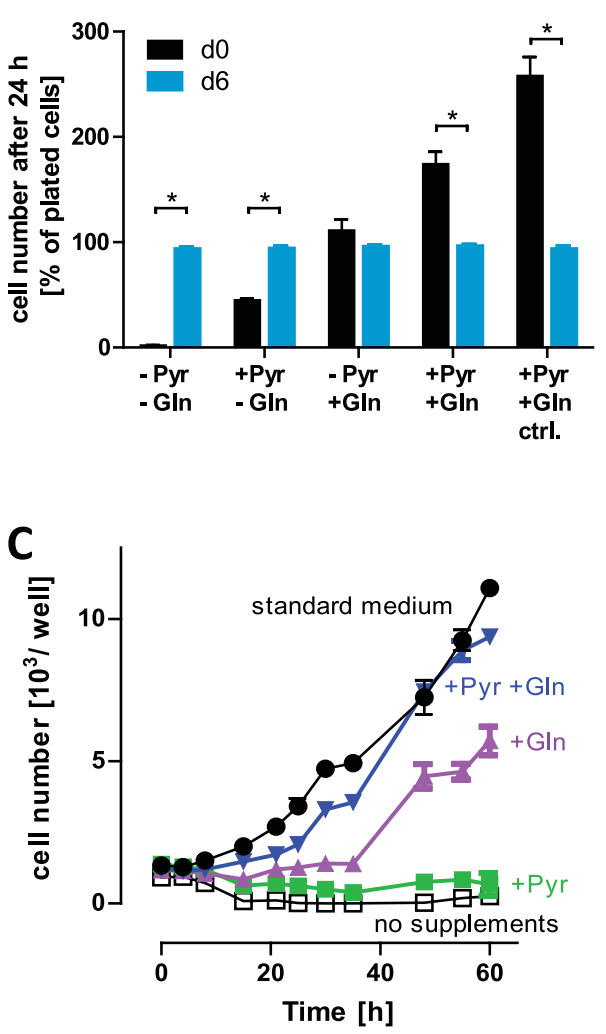

Fig. 7. Loss of glutamine dependency of LUHMES upon differentiation

LUHMES cells (d0 or d6) were cultured under standardized conditions. Various culture parameters were analyzed for up to 60 h, i.e. do cells (black) continuing proliferation for up to three days, and for $\mathrm{d} 6$ cells (blue) remaining in the state of differentiated neurons for up to $60 \mathrm{~h}$. A: Concentrations of glutamine (Gln), glutamate (Glu) and aspartate (Asp) in the cell culture medium. Measurements were performed for $0 \mathrm{~h}$ to $24 \mathrm{~h}$ in cultures of $\mathrm{d} 0$ and $\mathrm{d} 6$ cells. B + C: Cultures of $\mathrm{d} 0$ and $\mathrm{d} 6$ were prepared so that they contained similar and standardized numbers of cells at the time point when the experiment was initiated. Then, the medium was changed and cell numbers were recorded for the following $60 \mathrm{~h}$. The medium contained either no supplements or combinations of pyruvate $[1 \mathrm{mM}]$ and $\mathrm{L}$ glutamine $[2 \mathrm{mM}]$. As control, the standard Adv. DMEM/ F12 medium (ctrl.) with all supplements added by the commercial supplier was used. The cell number was assessed $24 \mathrm{~h}$ after medium change and expressed as percent of the amount of seeded cells at $t=0$. Alternatively, the absolute cell count was quantified by automated live cell microscopy over a $60 \mathrm{~h}$ time period. The number of cells per well is indicated. All data are means \pm SD from three biological replicates. *: $\mathrm{p}<0.05$, by one-way ANOVA followed by Dunnett's post-hoc test. (For interpretation of the references to color in this figure legend, the reader is referred to the web version of this article.) proliferation, while combination of glutamine and pyruvate allowed a doubling of the cell number (Fig. 7B). These results point to a crucial importance of pyruvate for proliferation in do cells although the pyruvate uptake rate was only about $10 \%$ of the glucose uptake rate $\left(29 \pm 8 \mathrm{nmol} / 10^{6} \mathrm{cells} / \mathrm{h}\right.$, Suppl. Table 2$)$.

In the following, the differential effects of medium supplements were studied for d0 LUHMES cells over a period of $60 \mathrm{~h}$. This series of experiments confirmed that LUHMES cells could not be maintained alive in medium lacking glutamine. Moreover, it was confirmed that the presence of pyruvate enhanced the effect of glutamine (a significant further increase in proliferation), while pyruvate alone had no effect (Fig. 7C).

These data suggested that glutaminolysis (i.e. uptake of glutamine and feeding into the TCA via glutamate to aKG) is a pivotal cellular metabolic process that is necessary for the proliferation of neural precursor cells by supplying carbon backbones for anaplerotic processes, such as building block synthesis, while pyruvate may support this process by acting as electron-sink (Fig. 4I) (Birsoy et al., 2015; Sullivan et al., 2015).

\subsection{Differential sensitivity of do vs. d6 cells for metabolic toxicants}

This study found by several approaches that d0 cells differ particularly from d6 cells in their limited capacity to upregulate mitochondrial metabolism and in their dependency on glutamine. The latter requires the mitochondrial TCA to be channeled into various metabolic pathways. Therefore, the question raised, whether this metabolic situation might sensitize d0 cells to mitochondrial toxicants. To obtain experimental data, the toxicity of antimycin A (ETC complex III inhibitor), rotenone (ETC complex I inhibitor) and UK-5099 (inhibitor of mitochondrial pyruvate transporter) was assessed by measuring resazurin reduction (viability assay) and lactate dehydrogenase (LDH) release (cell death assay). Generally, both assays give similar data on toxicant potency. The comparison of the EC50 values for $\mathrm{d} 0$ and $\mathrm{d} 6$ cells showed that proliferating cells were more sensitive to toxicants inhibiting mitochondrial metabolism. To get an indication on whether the cell-stage dependent toxicity was related to the energetic flexibility of the cells, we measured lactate release in the presence of rotenone. As expected, the $\mathrm{d} 6$ cells increased this parameter (a measure of glycolytic activity) by $100 \%$ in the presence of the toxicant, while d0 cells showed little adaptation (data not shown).

In a next step, it was tested whether d0 cells were generally more vulnerable. Thus, both cell stages were exposed to 3-bromopyruvate, a toxicant that affects energy metabolism not on the stage of mitochondria, but at glycolysis. In this experiment, d6 cells proved to be more sensitive than d0 cells (Fig. 8). For two non-specific toxicants, the proteasome inhibitor MG-132 and thiol-reactive reagent thiomersal, both cell stages showed similar sensitivities (data not shown).

\section{Discussion}

In this study, we linked key observations on metabolic changes occurring during neuronal differentiation to different stage specific toxicological sensitivities. Using multiple analytical approaches, including p-SRIM followed by metabolic flux modeling (INST ${ }^{13} \mathrm{C}-\mathrm{MFA}$ ), we found that the strong metabolic changes upon differentiation are mainly due to the changing metabolic needs of the cells undergoing differentiation from proliferating precursors to mature neurons. Proliferative cells were found to be particularly sensitive to toxicants that challenge the cellular metabolic buffering capacity. Since their metabolic spare capacities were minor, metabolic insults could not be compensated, e.g. by increased glycolysis. Previous approaches in toxicology have already used the combination of two or three omics techniques to attempt a system-wide characterization of cells and of a specific stress response after toxicant exposure (Carreras Puigvert et al., 2013; Wilmes et al., 2013; Krug et al., 2014; Wilmes et al., 2015). This 


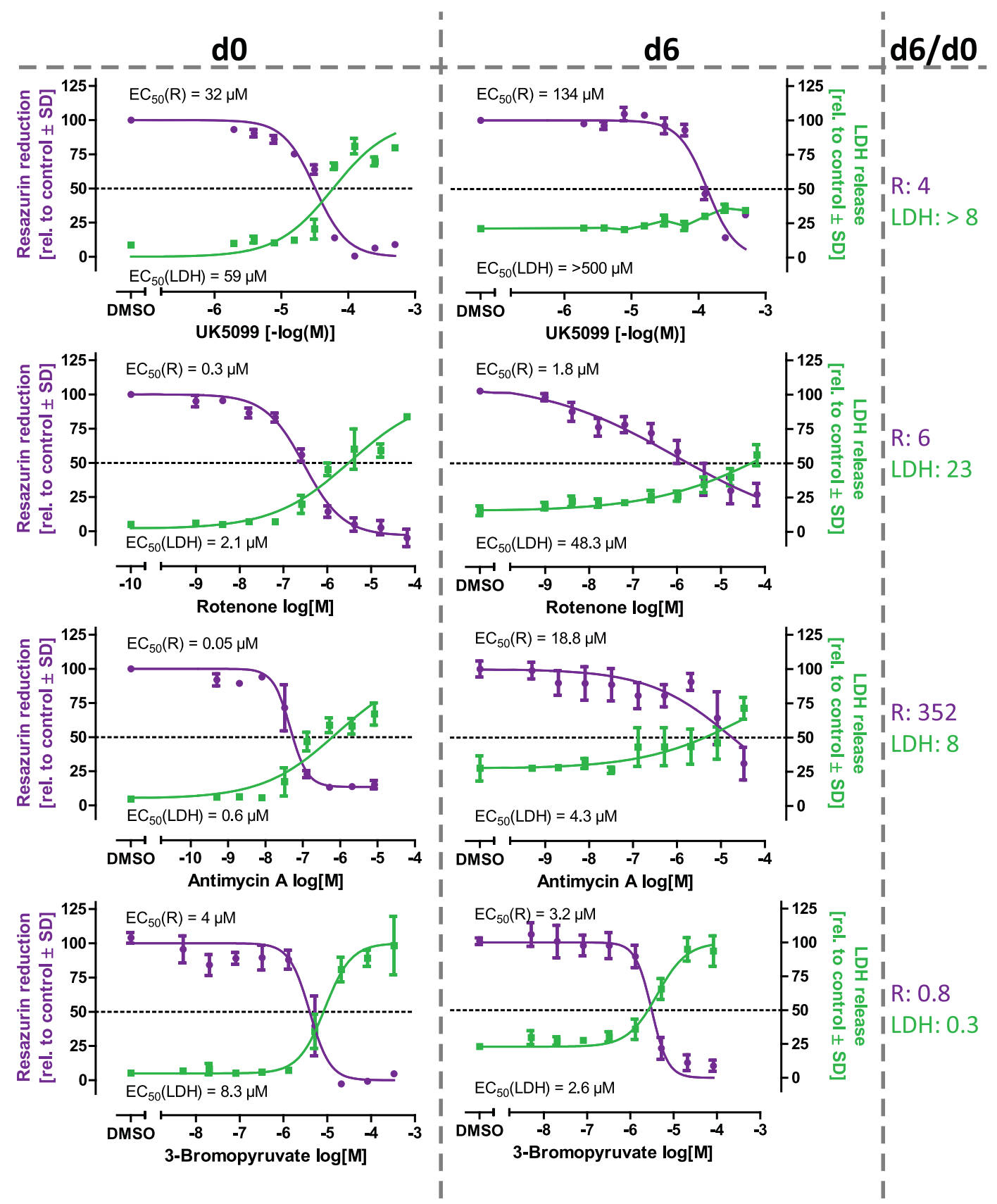

Fig. 8. Differential sensitivity to toxicants of differentiated neurons versus immature precursors

Proliferating (d0) or differentiated (d6) LUHMES neurons were used in 96-well plates to determine the cytotoxicity of mitochondrial inhibitors and of toxicants not inhibiting mitochondrial complexes. After $24 \mathrm{~h}$ exposure to serial dilutions of toxicants, resazurin reduction (R, purple) and lactate dehydrogenase release (LDH, green) were determined. Log-logistic curves were fit to the data, and these were used to determine $\mathrm{EC}_{50}$ values. The right hand column (d6/d0) indicates ratios for either $\mathrm{R}$ or $\mathrm{LDH}_{\mathrm{H}}$. They were derived by dividing the $\mathrm{EC}_{50}$ value of differentiated cells (d6) by the $\mathrm{EC}_{50}$ value of the progenitor cells (d0), respectively. All data are means \pm SD from three independent experiments. (For interpretation of the references to color in this figure legend, the reader is referred to the web version of this article.)

study contributed a comprehensive multi-omics approach comprising transcriptomics, proteomics, metabolomics, and fluxomics technologies. Two major insights were: (1) an extreme glutamine dependence of neural stem cells (d0) and (2) cell stage-specific neurotoxicity.

Metabolic states in LUHMES cells were inaccessible for traditional ${ }^{13} \mathrm{C}$-MFA relying on steady-state labeling enrichment due to long label incorporation times. Therefore, INST ${ }^{13} \mathrm{C}$-MFA with shortened labeling periods and time-resolved observations was implemented (Noh et al., 2006; Wiechert and Noh, 2013). This approach made use of the metabolomics data and allowed resolving the contribution of key metabolic pathways by joint inference of metabolite pool sizes, cell-specific extracellular rates and labeling incorporation profiles.
The first major observation of our study was the high dependency of neuronal precursor cells on extracellular glutamine. It is used for anaplerotic reactions of the citric acid cycle, when products of the cycle are used as cellular building blocks. In addition, glutamine is known to be a donor of amino-groups for non-essential amino acids as serine and glycine, which are also needed for building block and protein synthesis (Hensley et al., 2013). Furthermore, glutamine is a precursor of glutathione (via prior conversion to glutamate) and might thereby aid the maintenance of the cells' antioxidative potential and redox homeostasis (Hensley et al., 2013; Cetinbas et al., 2016). A recent publication found that proliferating cells, especially during S-phase, rely on glutamine anaplerosis of the TCA (Ahn et al., 2017), which is in line with d0 
LUHMES showing a high dependence on glutamine.

D0 LUHMES may fuel their TCA from glutamine, also in order to produce aspartate, as it has been described for cancer cells (Vander Heiden et al., 2009). Such aspartate production has been shown for tumor cell lines to be limiting for purine and pyrimidine synthesis (Birsoy et al., 2015; Sauer et al., 2015), as well as for NADPH production and maintenance of mitochondrial membrane potential and integrity (Wise and Thompson, 2010). Additionally, glutamine can be metabolized to AcCoA without generation of NADH and thereby not further burdening cells' mitochondria (Markert and Vazquez, 2015; Fernandez-de-Cossio-Diaz and Vazquez, 2017). Differentiated d6 cells have much less need for nucleotide synthesis and, therefore, require less glutamine and aspartate. Still these cells maintain a high non-growth related metabolic activity. Label incorporation profiles indicate that mitochondrial metabolism might be enhanced in $\mathrm{d} 6$ compared to $\mathrm{d} 0$ cells, but further comparative ${ }^{13} \mathrm{C}-\mathrm{MFA}$ studies are required to finally answer this question. Being successfully applied to a test system, this study provides an important basis for probing specific metabolic states for future studies on the mode of action of toxicants. Ultimately, mechanistic models of CCM will be needed to study these modes of action (Vasilakou et al., 2016) and to predict toxicological properties of unknown compounds (Bouhifd et al., 2013; Ramirez et al., 2013; Sturla et al., 2014; Sauer et al., 2015).

A potential flaw of the model we use is the expression of the myc transgene to maintain neural precursor state. We cannot exclude that myc also affects the metabolism, but there are several lines of evidence that suggest that this is a rather minor effect: (i) while we performed our study, a similar study was published on the basis iPSC-derived neurons (without myc transgene). The findings of this study (although related more to proteome changes) are quite similar to ours (Zheng et al., 2016); (ii) the expression levels of v-myc in LUHMES are quite moderate compared to endogenous c-myc and we found that the protein amounts were about similar; (iii) neural stem cells/neural crest cells do naturally express myc, and it plays an important role at this stage (Garcia-Castro et al., 2002; Bellmeyer et al., 2003; Nagao et al., 2008; Varlakhanova et al., 2010); (iv) it is known for some other cells that overexpression of myc (cancer environment) caused minimal changes in glutamine and glucose consumption, although spare respiratory capacity was increased (Anso et al., 2013).

Second, in the field of DNT, there has been a strong focus on fundamental neurodevelopmental processes as basis for test systems and assays (Bal-Price et al., 2015; Aschner et al., 2017). Here, we show that also different susceptibilities of a given cell differentiation stage can be important as target of developmental neurotoxicants. Our data suggest a link between the metabolic switch during differentiation and alterations in the sensitivity to toxicants. The lack of energetic buffering capacity, both mitochondrial and glycolytic, of proliferating d0 cells accounts for their higher sensitivity to UK5099, rotenone and antimycin. Instead, the $\mathrm{d} 6$ neurons were able to increase the glycolytic activity to counteract the mitochondrial inhibition, and therefore they showed higher tolerance to those toxicants. There are several other cases known, in which stage-specific toxicity occurs, e.g. exposure to the cell cycle toxicant methylazoxymethylacetat (MAM) (Penschuck et al., 2006). However, it should not be concluded from this example that proliferating cells are generally more sensitive to toxicants. Other literature data show that differentiated LUHMES cells are more susceptible to the toxicants colchicine, vincristine and methylmercury (Tong et al., 2017). A differential toxicity is not only observed for specific neurotoxicants, but it was also found for the glycolytic inhibitor 3bromopyruvate, for which d6 cells were twice as sensitive as their proliferating counterparts. These data suggest that mature neurons may on the one hand compensate certain metabolic stresses (e.g. partial mitochondrial inhibition), but they succumb more readily to the complete shut-down of energy production by a glycolytic inhibitor. The data from LUHMES cells on glycolytic compensation for mitochondrial inhibition are well in agreement with data from primary neurons
(Volbracht et al., 1999) and e.g. lymphocytes (Nicotera et al., 1999; Hirt et al., 2000). It also needs to be noted that our study did not address activity-dependent energy consumption of cells. For instance some adult neuronal populations, like nigrostriatal dopaminergic neurons, are particularly sensitive to mitochondrial inhibitors. The reason for this is the extremely high energy demand of these cells, when they are synaptically integrated (Schildknecht et al., 2017). This example shows, that metabolic balance may depend not only on basic biochemical features of cells (as studied here), but also on their cellular context. Ways to mimic this would be co-cultures (Efremova et al., 2015; Efremova et al., 2017) or 3D-cultures (Smirnova et al., 2016), and applying metabolomics to such advance models is an additional future challenge.

In summary, our study shows that a thorough characterization of the test system can be helpful, or sometimes is even pivotal for the understanding of toxicological observations and for the interpretation of their relevance. By using an approach combining classical omics techniques with ${ }^{13} \mathrm{C}-\mathrm{MFA}$ and cellular respiration measurements, we identified metabolic differences that accounted for changes in toxicant sensitivity. In the future this approach will be refined and expanded towards the description of toxicant effects in the nervous system. Key metabolic pathways have to be identified and mapped for various standard toxicants. This knowledge could allow for more targeted measurements at lower cost. By focusing on few pathways, metabolites and time points, the procedure could reach a throughput allowing broader screening (Buescher et al., 2015). As a hit-follow-up of the screening, a full mechanistic characterization may be added. This approach may identify cell stage-specific toxicants not easily detected by assays focusing on developmental processes. Since the loss of a highly proliferative subpopulation during neuro-development might result in a pronounced effect in later life, cell death of the proliferative precursors can be as fatal as impairment of migration (Nyffeler et al., 2017), neurite outgrowth (Krug et al., 2013a) or neuronal network formation (Brown et al., 2017; Pamies et al., 2017).

\section{Conflict of interest}

The authors declare no conflict of interest.

\section{Acknowledgements}

This work was supported by the DFG (RTG1331), the Land-BW (BW15986612/INVITE), the BMBF (BMBF31A271) and the EC project EU-ToxRisk (EC681002).

\section{References}

Agostini, M., Romeo, F., et al., 2016. Metabolic reprogramming during neuronal differentiation. Cell Death Differ. 23 (9), 1502-1514.

Ahn, W.S., Antoniewicz, M.R., 2011. Metabolic flux analysis of CHO cells at growth and non-growth phases using isotopic tracers and mass spectrometry. Metab. Eng. 13 (5), 598-609.

Ahn, W.S., Antoniewicz, M.R., 2013. Parallel labeling experiments with [1,2-(13)C]glucose and [U-(13)C]glutamine provide new insights into CHO cell metabolism. Metab. Eng. 15, 34-47.

Ahn, E., Kumar, P., et al., 2017. Temporal fluxomics reveals oscillations in TCA cycle flux throughout the mammalian cell cycle. Mol. Syst. Biol. 13 (11), 953.

Anso, E., Mullen, A.R., et al., 2013. Metabolic changes in cancer cells upon suppression of MYC. Cancer Metab. 1 (1), 7.

Aschner, M., Ceccatelli, S., et al., 2017. Reference compounds for alternative test methods to indicate developmental neurotoxicity (DNT) potential of chemicals: example lists and criteria for their selection and use. ALTEX 34 (1), 49-74.

Balmer, N.V., Weng, M.K., et al., 2012. Epigenetic changes and disturbed neural development in a human embryonic stem cell-based model relating to the fetal valproate syndrome. Hum. Mol. Genet. 21 (18), 4104-4114.

Balmer, N.V., Klima, S., et al., 2014. From transient transcriptome responses to disturbed neurodevelopment: role of histone acetylation and methylation as epigenetic switch between reversible and irreversible drug effects. Arch. Toxicol. 88 (7), 1451-1468. Bal-Price, A., Crofton, K.M., et al., 2015. International STakeholder NETwork (ISTNET): 
creating a developmental neurotoxicity (DNT) testing road map for regulatory purposes. Arch. Toxicol. 89 (2), 269-287.

Bellmeyer, A., Krase, J., et al., 2003. The protooncogene c-myc is an essential regulator of neural crest formation in xenopus. Dev. Cell 4 (6), 827-839.

Birsoy, K., Wang, T., et al., 2015. An essential role of the mitochondrial electron transport chain in cell proliferation is to enable aspartate synthesis. Cell 162 (3), 540-551.

Bouhifd, M., Hartung, T., et al., 2013. Review: toxicometabolomics. J. Appl. Toxicol. 33 (12), 1365-1383.

Brown, J.P., Lynch, B.S., et al., 2017. Assaying spontaneous network activity and cellular viability using multi-well microelectrode arrays. Methods Mol. Biol. 1601, 153-170.

Buescher, J.M., Antoniewicz, M.R., et al., 2015. A roadmap for interpreting (13)C metabolite labeling patterns from cells. Curr. Opin. Biotechnol. 34, 189-201.

Cabre, R., Jove, M., et al., 2016. Specific metabolomics adaptations define a differential regional vulnerability in the adult human cerebral cortex. Front. Mol. Neurosci. 9 (138).

Carinhas, N., Pais, D.A., et al., 2016. Metabolic flux profiling of MDCK cells during growth and canine adenovirus vector production. Sci. Rep. 6 (23529).

Carreras Puigvert, J., von Stechow, L., et al., 2013. Systems biology approach identifies the kinase Csnk1a1 as a regulator of the DNA damage response in embryonic stem cells. Sci. Signal. 6 (259) (ra5).

Cetinbas, N.M., Sudderth, J., et al., 2016. Glucose-dependent anaplerosis in cancer cells is required for cellular redox balance in the absence of glutamine. Sci. Rep. 6 (32606).

Croft, D., Mundo, A.F., et al., 2014. The Reactome pathway knowledgebase. Nucleic Acids Res. 42 (Database issue), D472-477.

Dreser, N., Zimmer, B., et al., 2015. Grouping of histone deacetylase inhibitors and other toxicants disturbing neural crest migration by transcriptional profiling. Neurotoxicology 50, 56-70.

Duarte, N.C., Becker, S.A., et al., 2007. Global reconstruction of the human metabolic network based on genomic and bibliomic data. Proc. Natl. Acad. Sci. U. S. A. 104 (6), $1777-1782$.

Efremova, L., Schildknecht, S., et al., 2015. Prevention of the degeneration of human dopaminergic neurons in an astrocyte co-culture system allowing endogenous drug metabolism. Br. J. Pharmacol. 172 (16), 4119-4132.

Efremova, L., Chovancova, P., et al., 2017. Switching from astrocytic neuroprotection to neurodegeneration by cytokine stimulation. Arch. Toxicol. 91 (1), 231-246.

Esteban-Martinez, L., Sierra-Filardi, E., et al., 2017. Programmed mitophagy is essential for the glycolytic switch during cell differentiation. EMBO J. 36 (12), 1688-1706.

Fabregat, A., Jupe, S., et al., 2017. The reactome pathway knowledgebase. Nucleic Acids Res.

Fan, J., Kamphorst, J.J., et al., 2013. Glutamine-driven oxidative phosphorylation is major ATP source in transformed mammalian cells in both normoxia and hypoxia. Mol. Syst. Biol. 9, 712.

Fernandez-de-Cossio-Diaz, J., Vazquez, A., 2017. Limits of aerobic metabolism in cancer cells. Sci. Rep. 7 (1), 13488

Folmes, C.D., Nelson, T.J., et al., 2011. Somatic oxidative bioenergetics transitions into pluripotency-dependent glycolysis to facilitate nuclear reprogramming. Cell Metab. 14 (2), 264-271.

Folmes, C.D., Dzeja, P.P., et al., 2012. Metabolic plasticity in stem cell homeostasis and differentiation. Cell Stem Cell 11 (5), 596-606.

Fritsche, E., Crofton, K.M., et al., 2017. OECD/EFSA workshop on developmental neurotoxicity (DNT): the use of non-animal test methods for regulatory purposes. ALTEX 34 (2), 311-315.

Garcia-Castro, M.I., Marcelle, C., et al., 2002. Ectodermal Wnt function as a neural crest inducer. Science 297 (5582), 848-851.

Gartlon, J., Kinsner, A., et al., 2006. Evaluation of a proposed in vitro test strategy using neuronal and non-neuronal cell systems for detecting neurotoxicity. Toxicol. in Vitro 20 (8), 1569-1581.

Haghighat, N., McCandless, D.W., 1997. Effect of 6-aminonicotinamide on metabolism of astrocytes and C6-glioma cells. Metab. Brain Dis. 12 (1), 29-45.

Hartung, T., Hoffmann, S., et al., 2013. Mechanistic validation. ALTEX 30 (2), 119-130.

Hensley, C.T., Wasti, A.T., et al., 2013. Glutamine and cancer: cell biology, physiology, and clinical opportunities. J. Clin. Invest. 123 (9), 3678-3684.

Hirt, U.A., Gantner, F., et al., 2000. Phagocytosis of nonapoptotic cells dying by caspaseindependent mechanisms. J. Immunol. 164 (12), 6520-6529.

Hu, X., Xuan, Y., 2008. Bypassing cancer drug resistance by activating multiple death pathways-a proposal from the study of circumventing cancer drug resistance by induction of necroptosis. Cancer Lett. 259 (2), 127-137.

Huang da, W., Sherman, B.T., et al., 2009a. Bioinformatics enrichment tools: paths toward the comprehensive functional analysis of large gene lists. Nucleic Acids Res. 37 (1), 1-13.

Huang da, W., Sherman, B.T., et al., 2009b. Systematic and integrative analysis of large gene lists using DAVID bioinformatics resources. Nat. Protoc. 4 (1), 44-57.

Jang, S., Nelson, J.C., et al., 2016. Glycolytic enzymes localize to synapses under energy stress to support synaptic function. Neuron 90 (2), 278-291.

Kadereit, S., Zimmer, B., et al., 2012. Compound selection for in vitro modeling of developmental neurotoxicity. Front. Biosci. (Landmark Ed) 17, 2442-2460.

Kahn, A., Meienhofer, M.C., et al., 1979. Phosphofructokinase (PFK) isozymes in man. I. Studies of adult human tissues. Hum. Genet. 48 (1), 93-108.

Kempa, S., Hummel, J., et al., 2009. An automated GCxGC-TOF-MS protocol for batchwise extraction and alignment of mass isotopomer matrixes from differential 13Clabelling experiments: a case study for photoautotrophic-mixotrophic grown Chlamydomonas reinhardtii cells. J. Basic Microbiol. 49 (1), 82-91.

Kleiderman, S., Gutbier, S., et al., 2016a. Conversion of nonproliferating astrocytes into neurogenic neural stem cells: control by FGF2 and interferon-gamma. Stem Cells 34 (12), 2861-2874

Kleiderman, S., Sa, J.V., et al., 2016b. Functional and phenotypic differences of pure populations of stem cell-derived astrocytes and neuronal precursor cells. Glia 64 (5), 695-715.

Krug, A.K., Balmer, N.V., et al., 2013a. Evaluation of a human neurite growth assay as specific screen for developmental neurotoxicants. Arch. Toxicol. 87 (12), 2215-2231.

Krug, A.K., Kolde, R., et al., 2013b. Human embryonic stem cell-derived test systems for developmental neurotoxicity: a transcriptomics approach. Arch. Toxicol. 87 (1), 123-143.

Krug, A.K., Gutbier, S., et al., 2014. Transcriptional and metabolic adaptation of human neurons to the mitochondrial toxicant MPP(+). Cell Death Dis. 5, e1222.

Kuich, P.H., Hoffmann, N., et al., 2014. Maui-VIA: a user-friendly software for visual identification, alignment, correction, and quantification of gas chromatography-mass spectrometry data. Front. Bioeng. Biotechnol. 2, 84.

Latta, M., Kunstle, G., et al., 2000. Metabolic depletion of ATP by fructose inversely controls CD95- and tumor necrosis factor receptor 1-mediated hepatic apoptosis. J. Exp. Med. 191 (11), 1975-1985.

Lau, C., Mole, M.L., et al., 2001. Toward a biologically based dose-response model for developmental toxicity of 5-fluorouracil in the rat: acquisition of experimental data. Toxicol. Sci. 59 (1), 37-48.

Leist, M., Bremer, S., et al., 2008a. The biological and ethical basis of the use of human embryonic stem cells for in vitro test systems or cell therapy. ALTEX 25 (3), 163-190.

Leist, M., Hartung, T., et al., 2008b. The dawning of a new age of toxicology. ALTEX 25 (2), 103-114

Leist, M., Lidbury, B.A., et al., 2012. Novel technologies and an overall strategy to allow hazard assessment and risk prediction of chemicals, cosmetics, and drugs with animal-free methods. ALTEX 29 (4), 373-388.

Lotharius, J., Falsig, J., et al., 2005. Progressive degeneration of human mesencephalic neuron-derived cells triggered by dopamine-dependent oxidative stress is dependent on the mixed-lineage kinase pathway. J. Neurosci. 25 (27), 6329-6342.

Magistretti, P.J., Allaman, I., 2015. A cellular perspective on brain energy metabolism and functional imaging. Neuron 86 (4), 883-901.

Markert, E.K., Vazquez, A., 2015. Mathematical models of cancer metabolism. Cancer Metab. 3 (14).

McKenna, M.C., 2007. The glutamate-glutamine cycle is not stoichiometric: fates of glutamate in brain. J. Neurosci. Res. 85 (15), 3347-3358.

Nagao, M., Campbell, K., et al., 2008. Coordinated control of self-renewal and differentiation of neural stem cells by Myc and the p19ARF-p53 pathway. J. Cell Biol. 183 (7), 1243-1257.

Nicolae, A., Wahrheit, J., et al., 2014. Non-stationary 13C metabolic flux analysis of Chinese hamster ovary cells in batch culture using extracellular labeling highlights metabolic reversibility and compartmentation. BMC Syst. Biol. 8, 50.

Nicotera, P., Leist, M., et al., 1999. Neuronal cell death: a demise with different shapes. Trends Pharmacol. Sci. 20 (2), 46-51.

Niedenfuhr, S., ten Pierick, A., et al., 2016. Natural isotope correction of MS/MS measurements for metabolomics and (13)C fluxomics. Biotechnol. Bioeng. 113 (5), $1137-1147$.

Ninomiya, Y., Adams, R., et al., 1997. Apoptotic cell death in neuronal differentiation of P19 EC cells: cell death follows reentry into S phase. J. Cell. Physiol. 172 (1), 25-35.

Noh, K., Wahl, A., et al., 2006. Computational tools for isotopically instationary 13C labeling experiments under metabolic steady state conditions. Metab. Eng. 8 (6), 554-577.

Noh, K., Droste, P., et al., 2015. Visual workflows for 13C-metabolic flux analysis. Bioinformatics 31 (3), 346-354.

Norton, W.T., Poduslo, S.E., 1971. Neuronal perikarya and astroglia of rat brain: chemical composition during myelination. J. Lipid Res. 12 (1), 84-90.

Nyffeler, J., Karreman, C., et al., 2017. Design of a high-throughput human neural crest cell migration assay to indicate potential developmental toxicants. ALTEX 34 (1), 75-94.

Pamies, D., Barreras, P., et al., 2017. A human brain microphysiological system derived from induced pluripotent stem cells to study neurological diseases and toxicity. ALTEX 34 (3), 362-376.

Penschuck, S., Flagstad, P., et al., 2006. Decrease in parvalbumin-expressing neurons in the hippocampus and increased phencyclidine-induced locomotor activity in the rat methylazoxymethanol (MAM) model of schizophrenia. Eur. J. Neurosci. 23 (1), 279-284.

Pietzke, M., Zasada, C., et al., 2014. Decoding the dynamics of cellular metabolism and the action of 3-bromopyruvate and 2-deoxyglucose using pulsed stable isotope-resolved metabolomics. Cancer Metab. 2, 9.

Poltl, D., Schildknecht, S., et al., 2012. Uncoupling of ATP-depletion and cell death in human dopaminergic neurons. Neurotoxicology 33 (4), 769-779.

Ramirez, T., Daneshian, M., et al., 2013. Metabolomics in toxicology and preclinical research. ALTEX 30 (2), 209-225.

Rempel, E., Hoelting, L., et al., 2015. A transcriptome-based classifier to identify developmental toxicants by stem cell testing: design, validation and optimization for histone deacetylase inhibitors. Arch. Toxicol. 89 (9), 1599-1618.

Sa, J.V., Kleiderman, S., et al., 2017. Quantification of metabolic rearrangements during neural stem cells differentiation into astrocytes by metabolic flux analysis. Neurochem. Res. 42 (1), 244-253.

Sauer, J.M., Hartung, T., et al., 2015. Systems toxicology: the future of risk assessment. Int. J. Toxicol. 34 (4), 346-348.

Schildknecht, S., Poltl, D., et al., 2009. Requirement of a dopaminergic neuronal phenotype for toxicity of low concentrations of 1-methyl-4-phenylpyridinium to human cells. Toxicol. Appl. Pharmacol. 241 (1), 23-35.

Schildknecht, S., Pape, R., et al., 2015. Preferential extracellular generation of the active parkinsonian toxin MPP + by transporter-independent export of the intermediate MPDP +. Antioxid. Redox Signal. 23 (13), 1001-1016.

Schildknecht, S., Di Monte, D.A., et al., 2017. Tipping points and endogenous 
determinants of nigrostriatal degeneration by MPTP. Trends Pharmacol. Sci. 38 (6), 541-555.

Schmidt, B.Z., Lehmann, M., et al., 2017. In vitro acute and developmental neurotoxicity screening: an overview of cellular platforms and high-throughput technical possibilities. Arch. Toxicol. 91 (1), 1-33.

Scholz, D., Poltl, D., et al., 2011. Rapid, complete and large-scale generation of postmitotic neurons from the human LUHMES cell line. J. Neurochem. 119 (5), 957-971.

Scholz, D., Chernyshova, Y., et al., 2013. Control of Abeta release from human neurons by differentiation status and RET signaling. Neurobiol. Aging 34 (1), 184-199.

Schuster, S., Boley, D., et al., 2015. Mathematical models for explaining the Warburg effect: a review focussed on ATP and biomass production. Biochem. Soc. Trans. 43 (6), 1187-1194.

Sengupta, S., Johnson, B.P., et al., 2014. Aggregate culture of human embryonic stem cellderived hepatocytes in suspension are an improved in vitro model for drug metabolism and toxicity testing. Toxicol. Sci. 140 (1), 236-245.

Sheikh, K., Forster, J., et al., 2005. Modeling hybridoma cell metabolism using a generic genome-scale metabolic model of Mus musculus. Biotechnol. Prog. 21 (1), 112-121.

Shinde, V., Klima, S., et al., 2015. Human pluripotent stem cell based developmental toxicity assays for chemical safety screening and systems biology data generation. J. Vis. Exp. 100, e52333.

Shinde, V., Perumal Srinivasan, S., et al., 2016. Comparison of a teratogenic transcriptome-based predictive test based on human embryonic versus inducible pluripotent stem cells. Stem Cell Res Ther 7 (1), 190.

Shinde, V., Hoelting, L., et al., 2017. Definition of transcriptome-based indices for quantitative characterization of chemically disturbed stem cell development: introduction of the STOP-Toxukn and STOP-Toxukk tests. Arch. Toxicol. 91 (2), 839-864.

Shyh-Chang, N., Daley, G.Q., et al., 2013. Stem cell metabolism in tissue development and aging. Development 140 (12), 2535-2547.

Smirnova, L., Hogberg, H.T., et al., 2014. Developmental neurotoxicity - challenges in the 21st century and in vitro opportunities. ALTEX 31 (2), 129-156.

Smirnova, L., Harris, G., et al., 2015. Cellular resilience. ALTEX 32 (4), 247-260.

Smirnova, L., Harris, G., et al., 2016. A LUHMES 3D dopaminergic neuronal model for neurotoxicity testing allowing long-term exposure and cellular resilience analysis. Arch. Toxicol. 90 (11), 2725-2743.

Smith, T.A., 2000. Mammalian hexokinases and their abnormal expression in cancer. Br. J. Biomed. Sci. 57 (2), 170-178.

Sturla, S.J., Boobis, A.R., et al., 2014. Systems toxicology: from basic research to risk assessment. Chem. Res. Toxicol. 27 (3), 314-329.

Sullivan, L.B., Gui, D.Y., et al., 2015. Supporting aspartate biosynthesis is an essential function of respiration in proliferating cells. Cell 162 (3), 552-563.

Swanson, R.A., Graham, S.H., 1994. Fluorocitrate and fluoroacetate effects on astrocyte metabolism in vitro. Brain Res. 664 (1-2), 94-100.

Teslaa, T., Teitell, M.A., 2015. Pluripotent stem cell energy metabolism: an update. EMBO J. 34 (2), 138-153.

Thomas, P.D., Campbell, M.J., et al., 2003. PANTHER: a library of protein families and subfamilies indexed by function. Genome Res. 13 (9), 2129-2141.

Thomas, P.D., Kejariwal, A, et al., 2006. Applications for protein sequence-function evolution data: mRNA/protein expression analysis and coding SNP scoring tools. Nucleic Acids Res. 34, W645-650 Web Server issue.

van Thriel, C., Westerink, R.H., et al., 2012. Translating neurobehavioural endpoints of developmental neurotoxicity tests into in vitro assays and readouts. Neurotoxicology 33 (4), 911-924.

Tong, Z.B., Hogberg, H., et al., 2017. Characterization of three human cell line models for high-throughput neuronal cytotoxicity screening. J. Appl. Toxicol. 37 (2), 167-180. Vander Heiden, M.G., Cantley, L.C., et al., 2009. Understanding the Warburg effect: the metabolic requirements of cell proliferation. Science 324 (5930), 1029-1033.

Varlakhanova, N.V., Cotterman, R.F., et al., 2010. myc maintains embryonic stem cell pluripotency and self-renewal. Differentiation 80 (1), 9-19.

Vasilakou, E., Machado, D., et al., 2016. Current state and challenges for dynamic metabolic modeling. Curr. Opin. Microbiol. 33, 97-104.

Vazquez, A., Liu, J., et al., 2010. Catabolic efficiency of aerobic glycolysis: the Warburg effect revisited. BMC Syst. Biol. 4, 58 .

Volbracht, C., Leist, M., et al., 1999. ATP controls neuronal apoptosis triggered by microtubule breakdown or potassium deprivation. Mol. Med. 5 (7), 477-489.

Volbracht, C., Leist, M., et al., 2001. Apoptosis in caspase-inhibited neurons. Mol. Med. 7 (1), 36-48.

Waldmann, T., Grinberg, M., et al., 2017. Stem cell transcriptome responses and corresponding biomarkers that indicate the transition from adaptive responses to cytotoxicity. Chem. Res. Toxicol. 30 (4), 905-922.

Weitzel, M., Noh, K., et al., 2013. 13CFLUX2-high-performance software suite for (13)Cmetabolic flux analysis. Bioinformatics 29 (1), 143-145.

Wiechert, W., Noh, K., 2013. Isotopically non-stationary metabolic flux analysis: complex yet highly informative. Curr. Opin. Biotechnol. 24 (6), 979-986.

Wilmes, A., Limonciel, A., et al., 2013. Application of integrated transcriptomic, proteomic and metabolomic profiling for the delineation of mechanisms of drug induced cell stress. J. Proteome 79, 180-194.

Wilmes, A., Bielow, C., et al., 2015. Mechanism of cisplatin proximal tubule toxicity revealed by integrating transcriptomics, proteomics, metabolomics and biokinetics. Toxicol. In Vitro 30 (1 Pt A), 117-127.

Wilson, J.E., 2003. Isozymes of mammalian hexokinase: structure, subcellular localization and metabolic function. J. Exp. Biol. 206 (Pt 12), 2049-2057.

Wise, D.R., Thompson, C.B., 2010. Glutamine addiction: a new therapeutic target in cancer. Trends Biochem. Sci. 35 (8), 427-433.

Zhang, J., Nuebel, E., et al., 2012. Metabolic regulation in pluripotent stem cells during reprogramming and self-renewal. Cell Stem Cell 11 (5), 589-595.

Zheng, X., Boyer, L., et al., 2016. Metabolic reprogramming during neuronal differentiation from aerobic glycolysis to neuronal oxidative phosphorylation. elife 5.

Zimmer, B., Lee, G., et al., 2012. Evaluation of developmental toxicants and signaling pathways in a functional test based on the migration of human neural crest cells. Environ. Health Perspect. 120 (8), 1116-1122. 